\title{
SN 2017gmr: An Energetic Type II-P Supernova with Asymmetries
}

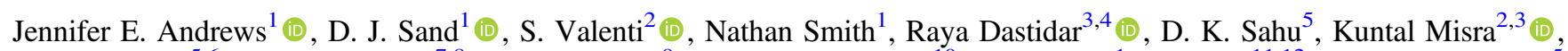

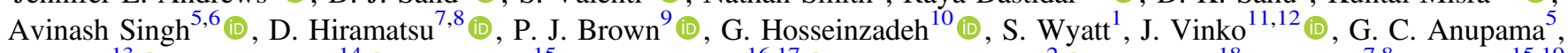
I. Arcavi $^{13}$ (1) Chris Ashall $^{14}$ (10), S. Benetti ${ }^{15}$, Marco Berton ${ }^{16,17}$ (1) K. A. Bostroem ${ }^{2}$ (D) M. Bulla ${ }^{18}$, J. Burke ${ }^{7,8}$, S. Chen ${ }^{15,19}$, L. Chomiuk ${ }^{20}$ (1) A. Cikota ${ }^{21}$, E. Congiu ${ }^{22,23}$, B. Cseh $^{11}$, Scott Davis ${ }^{14}$, N. Elias-Rosa ${ }^{24,25}$, T. Faran ${ }^{26}$, Morgan Fraser ${ }^{27}$ (1), L. Galbany ${ }^{28}$ (1) , C. Gall ${ }^{29}$ (1) A. Gal-Yam ${ }^{30}$ (D) Anjasha Gangopadhyay, ${ }^{3,31}$, M. Gromadzki ${ }^{32}$, J. Haislip ${ }^{33}$, D. A. Howell ${ }^{7,8}$ (D),

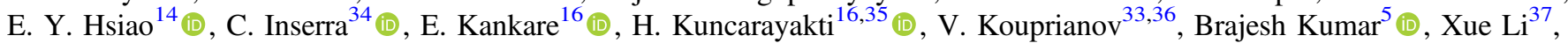

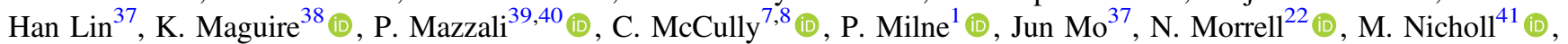

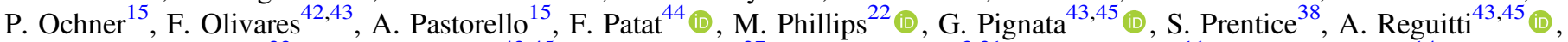
D. E. Reichart ${ }^{33}$ (1) Ó. Rodríguez ${ }^{43,45}$ (1) Liming Rui $^{37}$, Pankaj Sanwal ${ }^{3,31}$, K. Sárneczky ${ }^{11}$, M. Shahbandeh ${ }^{14}$ (1)

Mridweeka Singh ${ }^{3,31}$, S. Smartt ${ }^{46}$ (1) J. Strader ${ }^{20}$ (1) , M. D. Stritzinger ${ }^{47}$ (1) R. Szakáts ${ }^{11}$ (1) , L. Tartaglia ${ }^{48}$ (1), Huijuan Wang ${ }^{49}$, Lingzhi Wang ${ }^{49,50}$ (1) , Xiaofeng Wang ${ }^{37}$ (D) J. C. Wheeler ${ }^{12}$ (1) , Danfeng Xiang ${ }^{37}$, O. Yaron ${ }^{30}$, D. R. Young ${ }^{46}$ (1) and Junbo Zhang ${ }^{49}$

${ }^{1}$ Steward Observatory, University of Arizona, 933 North Cherry Avenue, Tucson, AZ 85721-0065, USA; jandrews@ as.arizona.edu

2 Department of Physics, University of California, 1 Shields Avenue, Davis, CA 95616-5270, USA

Aryabhatta Research Institute of Observational Sciences, Manora Peak, Nainital 263 001, India

${ }^{4}$ Department of Physics \& Astrophysics, University of Delhi, Delhi-110 007, India ${ }^{5}$ Indian Institute of Astrophysics, Koramangala, Bengaluru 560 034, India

${ }^{6}$ Joint Astronomy Programme, Department of Physics, Indian Institute of Science, Bengaluru 560012, India

${ }^{7}$ Department of Physics, University of California, Santa Barbara, CA 93106-9530, USA

${ }^{8}$ Las Cumbres Observatory, 6740 Cortona Drive, Suite 102, Goleta, CA 93117-5575, USA

${ }^{9}$ Mitchell Institute for Fundamental Physics and Astronomy, Texas A\&M University, College Station, TX 77843, USA

${ }^{10}$ Center for Astrophysics, Harvard \& Smithsonian, 60 Garden Street, Cambridge, MA 02138-1516, USA

${ }^{11}$ Konkoly Observatory, Research Centre for Astronomy and Earth Sciences, Hungarian Academy of Sciences, H-1121 Budapest, Hungary

${ }_{12}$ Department of Astronomy, University of Texas at Austin, Austin, TX 78712, USA

${ }^{13}$ The School of Physics and Astronomy, Tel Aviv University, Tel Aviv 69978, Israel

${ }^{14}$ Department of Physics, Florida State University, Tallahassee, FL 32306, USA

${ }_{15}$ INAF-Osservatorio Astronomico di Padova, Vicolo dell'Osservatorio 5, I-35122 Padova, Italy

${ }^{16}$ Tuorla Observatory, Department of Physics and Astronomy, University of Turku, FI-20014 Turku, Finland

${ }^{17}$ Aalto University Metsähovi Radio Observatory, Metsähovintie 114, FI-02540 Kylmälä, Finland

${ }^{18}$ The Oskar Klein Centre, Physics Department, Stockholm University, SE-106 91 Stockholm, Sweden

${ }^{19}$ Center for Astrophysics, Guangzhou University, Guangzhou 510006, People's Republic of China

${ }^{20}$ Department of Physics and Astronomy, Michigan State University, East Lansing, MI 48824, USA

${ }^{21}$ Physics Division, Lawrence Berkeley National Laboratory, 1 Cyclotron Road, Berkeley, CA 94720, USA

${ }^{22}$ Carnegie Observatories, Las Campanas Observatory, Casilla 601, La Serena, Chile

${ }^{23}$ INAF-Osservatorio Astronomico di Brera, via E. Bianchi 46, I-23807 Merate(LC), Italy

${ }^{24}$ Institute of Space Sciences (ICE, CSIC), Campus UAB, Carrer de Can Magrans s/n, E-08193 Barcelona, Spain

${ }^{25}$ Institut d'Estudis Espacials de Catalunya (IEEC), c/Gran Capitá 2-4, Edif. Nexus 201, E-08034 Barcelona, Spain

${ }^{26}$ Racah Institute of Physics, The Hebrew University of Jerusalem, Jerusalem 91904, Israel

${ }_{27}^{27}$ School of Physics, O’Brien Centre for Science North, University College Dublin, Belfield, Dublin 4, Ireland

${ }^{28}$ PITT PACC, Department of Physics and Astronomy, University of Pittsburgh, Pittsburgh, PA 15260, USA

${ }^{29}$ DARK, Niels Bohr Institute, University of Copenhagen, Lyngbyvej 2, DK-2100 Copenhagen, Denmark

${ }^{30}$ Department of Particle Phys., Astrophys., Weizmann Institute of Science, Rehovot 76100, Israel ${ }^{31}$ Pt. Ravi Shankar Shukla University, Raipur 492 010, India

${ }^{32}$ Warsaw University Astronomical Observatory, Al. Ujazdowskie 4, 00-478 Warszawa, Poland

${ }^{33}$ Warsaw University Astronomical Observatory, Al. Ujazdowskie 4, 00-478 Warszawa, Poland
Department of Physics and Astronomy, University of North Carolina at Chapel Hill, Chapel Hill, NC 27599, USA

${ }^{34}$ School of Physics and Astronomy, Cardiff University, Queens Buildings, The Parade, Cardiff, CF24 3AA, UK

${ }^{35}$ Finnish Centre for Astronomy with ESO (FINCA), University of Turku, Quantum, Vesilinnantie 5, FI-20014 University of Turku, Finland

${ }^{36}$ Central (Pulkovo) Observatory of Russian Academy of Sciences, 196140 Pulkovskoye Ave. 65/1, Saint Petersburg, Russia

${ }^{37}$ Physics Department and Tsinghua Center for Astrophysics (THCA), Tsinghua University, Beijing, 100084, People's Republic of China ${ }^{38}$ School of Physics, Trinity College Dublin, Dublin 2, Ireland

${ }^{39}$ Astrophysics Research Institute, Liverpool John Moores University, IC2, Liverpool Science Park, 146 Brownlow Hill, Liverpool L3 5RF, UK

${ }^{40}$ Max-Planck-Institut fur Astrophysik, Karl-Schwarzschild-Str. 1, D-85748 Garching, Germany

${ }^{41}$ Institute for Astronomy, University of Edinburgh, Royal Observatory, Blackford Hill, Edinburgh EH9 3HJ, UK

${ }_{42}$ Instituto de Astronomía y Ciencias Planetarias, Universidad de Atacama, Copayapu 485, Copiapó, Chile

${ }^{43}$ Millennium Institute of Astrophysics (MAS), Nuncio Monseñor Sótero Sanz 100, Providencia, Santiago, Chile

${ }^{44}$ European Southern Observatory, Karl-Schwarzschild-Str. 2, D-85748 Garching b. München, Germany

${ }^{45}$ Departamento de Ciencias Fisicas, Universidad Andres Bello, Avda. Republica 252, Santiago, Chile

${ }_{47}$ Astrophysics Research Centre, School of Mathematics and Physics, Queens University Belfast, BT7 1NN, UK

47 Department of Physics and Astronomy, Aarhus University, Ny Munkegade 120, DK-8000 Aarhus C, Denmark

${ }^{48}$ Department of Astronomy and The Oskar Klein Centre, AlbaNova University Centre, Stockholm University, SE-106 91 Stockholm, Sweden

${ }^{49}$ Key Laboratory of Optical Astronomy, National Astronomical Observatories, Chinese Academy of Sciences, Beijing 100101, People's Republic of China

${ }^{50}$ Chinese Academy of Sciences South America Center for Astronomy, China-Chile Joint Center for Astronomy, Camino El Observatorio \# 1515, Las Condes, Santiago, Chile

Received 2019 July 3; revised 2019 August 10; accepted 2019 August 31; published 2019 October 29 


\begin{abstract}
We present high-cadence UV, optical, and near-infrared data on the luminous Type II-P supernova SN 2017gmr from hours after discovery through the first 180 days. SN $2017 \mathrm{gmr}$ does not show signs of narrow, high-ionization emission lines in the early optical spectra, yet the optical light-curve evolution suggests that an extra energy source from circumstellar medium (CSM) interaction must be present for at least 2 days after explosion. Modeling of the early light curve indicates a $\sim 500 R_{\odot}$ progenitor radius, consistent with a rather compact red supergiant, and latetime luminosities indicate that up to $0.130 \pm 0.026 M_{\odot}$ of ${ }^{56} \mathrm{Ni}$ are present, if the light curve is solely powered by radioactive decay, although the ${ }^{56} \mathrm{Ni}$ mass may be lower if CSM interaction contributes to the post-plateau luminosity. Prominent multipeaked emission lines of $\mathrm{H} \alpha$ and [O I] emerge after day 154, as a result of either an asymmetric explosion or asymmetries in the CSM. The lack of narrow lines within the first 2 days of explosion in the likely presence of CSM interaction may be an example of close, dense, asymmetric CSM that is quickly enveloped by the spherical supernova ejecta.
\end{abstract}

Unified Astronomy Thesaurus concepts: Type II supernovae (1731); Core-collapse supernovae (304); Massive stars (732)

Supporting material: machine-readable table

\section{Introduction}

Core-collapse supernovae (CCSNe) mark the death of stars more massive than $\sim 8 M_{\odot}$. Those stars that end their lives with portions of their hydrogen envelope remaining are classified as Type II events (see Arcavi 2017; Branch \& Wheeler 2017; Gal-Yam 2017, for detailed reviews). Historically these events have been classified as Type II-P or Type II-L based on their light-curve shapes. Type II-P SNe ("P" for plateau) show a plateau phase of near-constant luminosity in the light curve for $\sim 2-3$ months after maximum light owing to the long diffusion and recombination timescales of the hydrogen envelope, while Type II-L SNe ("L" is for linear) show an almost linear decline with no or short plateau phases. Recent work has suggested that this bimodal classification is misleading, and in fact Type II SNe form a continuous class (Anderson et al. 2014; Galbany et al. 2016; Valenti et al. 2016). Once the recombination phase ends, a sharp drop in luminosity occurs over a relatively short timescale, until the SN settles into the nebular phase, where the light curve is powered primarily by radioactive decay.

Pre-explosion Hubble Space Telescope imaging of Type II-P events point to red supergiant (RSG) stars as the most common progenitors (Van Dyk et al. 2003; Smartt et al. 2009, 2015). RSGs do not form a homogeneous group, and variations in metallicity, initial mass, and mass-loss histories lead to diversity among the resultant SNe. Adopted mass-loss rates for RSGs generally range from $\sim 10^{-6}$ to $10^{-4} M_{\odot} \mathrm{yr}^{-1}$, with average wind velocities of $10 \mathrm{~km} \mathrm{~s}^{-1}$ (Mauron \& Josselin 2011; Goldman et al. 2017; Beasor \& Davies 2018). A recent study of early-time, high-cadence light curves in Förster et al. (2018) finds evidence for mass-loss rates greater than $10^{-4} M_{\odot}$ in the majority of their RSG sample. It is important to remember that these rates are for single-star models, and since $\sim 75 \%$ of massive stars in binaries have separations that can lead to interaction (Kiminki \& Kobulnicky 2012; Sana et al. 2012; de Mink et al. 2014; Moe \& Di Stefano 2017), mass-loss rates and densities could vary if a companion is present.

In $\sim 8 \%-9 \%$ of CCSNe the circumstellar medium (CSM) surrounding the progenitor is photoionized or shock heated, creating narrow $\left(\sim 100 \mathrm{~km} \mathrm{~s}^{-1}\right)$ hydrogen emission lines in their spectra (Smith et al. 2011a). The narrow lines lend themselves to the name Type IIn, where the " $n$ " stands for narrow (Schlegel 1990). The progenitors of these Type IIn SNe are likely special cases of evolved massive stars with pre-SN outbursts and could include RSGs, yellow hypergiants (YHGs), or luminous blue variables (LBVs) (Gal-Yam et al. 2007; GalYam \& Leonard 2009; Smith 2014).

If an SN is observed early enough, before the SN ejecta overtake the surrounding material, narrow lines from slow CSM can be detected in otherwise normal SNe (Niemela et al. 1985; Benetti et al. 1994; Leonard et al. 2000; Quimby et al. 2007). If present, these early and brief spectral features can be used to infer properties about the progenitor star such as massloss history and composition (Gal-Yam et al. 2014; Groh et al. 2014; Davies \& Dessart 2019). Additionally, if the CSM is dense enough, shock interaction with the SN ejecta can occur, converting the kinetic energy of the fast ejecta to radiative energy, thus increasing the luminosity of the SN. All of these features disappear within a week of explosion, eliminating them from the traditional class of Type IIn SNe. To date, only a handful of objects have shown these early high-ionization narrow emission lines, including SN 2013cu (Gal-Yam et al. 2014), SN 1998S (Shivvers et al. 2015), PTF1 1iqb (Smith et al. 2015), SN 2013fs (Yaron et al. 2017; Bullivant et al. 2018), and SN 2016bkv (Hosseinzadeh et al. 2018; Nakaoka et al. 2018). Others have shown a featureless, blue continuum with no lines (Khazov et al. 2016). As we discuss below, SN 2017gmr was observed within 1.5 days of explosion and showed no signs of narrow emission other than $\mathrm{H} \alpha$ in early spectroscopy.

SN $2017 \mathrm{gmr}$ was discovered at R.A. $(2000)=02^{\mathrm{h}} 35^{\mathrm{m}} 30^{\mathrm{s}} \cdot 15$, decl.(2000) $=-09^{\circ} 21^{\prime} 14^{\prime \prime} 95$ during the course of the DLT40 1-day cadence SN search (see Tartaglia et al. 2018, for a description of the survey) in the northeastern portion of NGC 988 (Figure 1) on 2017 September 4.25 UT (Valenti et al. 2017, MJD 58,000.266); it was given the designation DLT17cq by the DLT40 team, but we use the IAU naming convention and refer to it as SN $2017 \mathrm{gmr}$ throughout this work. The discovery magnitude was $r=15.12\left(M_{r} \approx-16.3\right.$, given the distance modulus we adopt below), and DLT40 observations taken 2 days prior to discovery (MJD 57,998.230) show no source at the position of the transient down to $r \gtrsim 19.4$ mag $\left(M_{r} \gtrsim-12.1\right)$, indicating that the SN was caught very close to the time of explosion. In Section 4 below we model the early-time light curves to constrain the explosion time and settle on MJD 57,999.09 (2017 September 3.08) as the epoch of explosion, and we adopt this value throughout the paper.

Spectroscopic observations conducted on 2017 September 6.19 allowed classification of this object as a possible core-collapse SN 


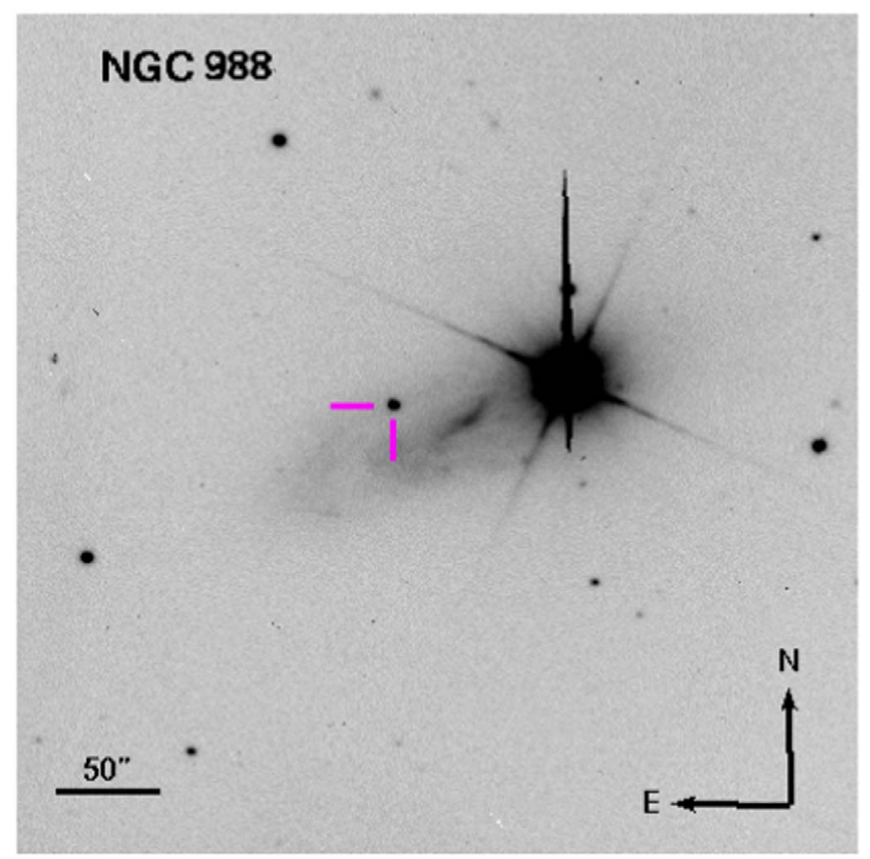

Figure 1. SN 2017gmr in NGC 988 taken on 2017 November 25 in $V$ band with Super-LOTIS. Image is $7^{\prime} \times 7^{\prime}$.

(Pursimo et al. 2017); it was confirmed as a Type II with broad Balmer lines in emission and moderate reddening about 1 week after explosion, on 2017 September 10.2 (Elias-Rosa et al. 2017). Adopting a redshift of $z=0.00504$ (Koribalski et al. 2004), an $H_{0}=73.5 \mathrm{~km} \mathrm{~s}^{-1} \mathrm{Mpc}^{-1}$ (Riess et al. 2018), and the Virgo infall velocity for the host NGC 988 given by NASA/IPAC Extragalactic Database (NED), $v_{\text {Virgo }}=1438 \pm 8 \mathrm{~km} \mathrm{~s}^{-1}$, we obtain a $\mu=31.46 \pm 0.15 \mathrm{mag}$, or a distance of $19.6 \pm 1.4 \mathrm{Mpc}$. NGC 988 is located in the same group as NGC 1084, the host galaxy of SN 2012ec, whose distance modulus was determined to be $\mu=31.36 \pm 0.15 \mathrm{mag}$ in Rodríguez et al. (2019), bolstering our confidence in the assumed distance. If we instead use the $3 \mathrm{~K}$ $\mathrm{CMB}$ velocity $v_{\mathrm{CMB}}=1288 \pm 16 \mathrm{~km} \mathrm{~s}^{-1}$ or the Local Group velocity $v_{\mathrm{LG}}=1532 \pm 5 \mathrm{~km} \mathrm{~s}^{-1}$, this changes the distance to $17.5 \pm 1.2 \mathrm{Mpc}$ or $20.8 \pm 1.5$, respectively. The Virgo infall values are more consistent with our host galaxy line measurements and fall nicely within other cosmological distance measurements, so we will use that value throughout the paper.

The paper is structured as follows: in Section 2 observations and data reduction are outlined, the reddening estimation is presented in Section 3, in Section 4 we discuss the optical and IR photometric evolution, Section 5 details the spectroscopic evolution of the object, in Section 6 we lay out the implications of the observational data, and finally the results are summarized in Section 7.

\section{Observations}

A comprehensive optical and near-infrared (NIR) data set has been collected on SN 2017gmr, with several major SN collaborations contributing data. These include the Las Cumbres Observatory's Global Supernova Project (e.g., Szalai et al. 2019), the NOT (Nordic Optical Telescope) Un-biased Transient Survey ${ }^{51}$ (NUTS), the Public ESO Spectroscopic Survey for Transient Objects (ePESSTO; Smartt et al. 2015),

\footnotetext{
51 http://csp2.1co.cl/not/
}

the Texas Supernova Spectroscopic Survey $\left(\mathrm{TS}^{3}\right)$, and the Tsinghua Supernova Project (X. Wang et al. 2019, in preparation). Below we briefly list the instruments/telescopes used in obtaining data for SN $2017 \mathrm{gmr}$, but for ease of reading an accounting of reduction procedures is included in Appendices A and B.

Continued photometric monitoring of SN 2017gmr was done by the DLT40 survey's two discovery telescopes, the PROMPT5 $0.4 \mathrm{~m}$ telescope at Cerro Tololo International Observatory and the PROMPT-MO $0.4 \mathrm{~m}$ telescope at Meckering Observatory in Australia, operated by the Skynet telescope network (Reichart et al. 2005). Additionally, an intense photometric campaign by the Las Cumbres Observatory telescope network (Brown et al. 2013), under the auspices of the Global Supernova Project, was begun immediately after discovery, in the UBVgri bands. Photometric data points were also taken at (1) the $0.6 \mathrm{~m}$ Schmidt telescope at Konkoly Observatory in the BVRI bands; (2) the $0.6 \mathrm{~m}$ Super-LOTIS telescope at Kitt Peak in the BVRI bands; (3) the $2.0 \mathrm{~m}$ Liverpool Telescope and the Optical Wide Field camera (IO:O) in the BVugriz bands; (4) the $2.56 \mathrm{~m}$ NOT Alhambra Faint Object Spectrograph and Camera (ALFOSC) in the BVugriz bands; (5) the Asiago Schmidt 67/92 cm telescope in the BVgri bands; (6) the $1.04 \mathrm{~m}$ Sampurnanand Telescope (ST) at Manora Peak, Nainital, in BVRI bands (Sagar 1999); (7) the $1.30 \mathrm{~m}$ Devasthal Fast Optical Telescope (DFOT) at Devasthal, Nainital, in UBVRIgri bands (Sagar et al. 2012); (8) the $2.01 \mathrm{~m}$ Himalayan Chandra Telescope (HCT) at Indian Astronomical Observatory (IAO) in Hanle, India (Prabhu \& Anupama 2010), in the UBVRI bands; (9) the $60 \mathrm{~cm}$ REM telescope in griz; and (10) the Tsinghua-NAOC $0.8 \mathrm{~m}$ telescope (Huang et al. 2012) in BVgri bands. Neil Gehrels Swift Observatory (Gehrels et al. 2004; Swift) UV and optical imaging was obtained of the early portion of the light curve. Furthermore, NIR $J, H$, and $K_{s}$ images were taken with NOTCam on the $2.56 \mathrm{~m}$ NOT telescope and the REM $60 \mathrm{~cm}$ telescope.

Many optical spectra were taken with the robotic FLOYDS spectrographs on the $2 \mathrm{~m}$ Faulkes Telescope North and South (FTN and FTS; Brown et al. 2013). Other telescopes/ instruments used were (1) the Goodman spectrograph (Clemens et al. 2004) on the $4.1 \mathrm{~m}$ SOAR telescope, (2) the Intermediate Dispersion Spectrograph (IDS) on the $2.54 \mathrm{~m}$ Isaac Newton Telescope (INT), (3) the Inamori-Magellan Areal Camera \& Spectrograph (IMACS; Dressler et al. 2011) on the $6.5 \mathrm{~m}$ Magellan Baade telescope, (4) the ALFOSC spectrograph on NOT, (5) the ESO Faint Object Spectrograph and Camera 2 (EFOSC2) on the $3.58 \mathrm{~m}$ New Technology Telescope (NTT), (6) the Beijing Faint Object Spectrograph and Camera (BFOSC) on the Xinglong $2.16 \mathrm{~m}$ telescope, (7) the Asiago Faint Object Spectrograph and Camera (AFOSC) on the Asiago $1.82 \mathrm{~m}$ telescope, (8) the FOcal Reducer and low dispersion Spectrograph 2 (FORS2; Appenzeller et al. 1998) on the $8.2 \mathrm{~m}$ Very Large Telescope (VLT), (9) the Himalaya Faint Object Spectrograph and Camera (HFOSC) on HCT, (10) the Boller \& Chivens (B\&C) Spectrograph mounted on the Asiago $1.22 \mathrm{~m}$ telescope, (11) the Low Resolution Spectrograph (LRS2; Chonis et al. 2016) on the effective $10 \mathrm{~m}$ Hobby-Eberly Telescope (HET), and (12) the Boller \& Chivens (B\&C) Spectrograph mounted on the $2.3 \mathrm{~m}$ Bok telescope on Kitt Peak. Further, a moderate-resolution spectrum was obtained with the Blue Channel (BC) spectrograph on the $6.5 \mathrm{~m} \mathrm{MMT.}$ 
High-resolution echelle spectra were taken with the HIghResolution Echelle Spectrograph (HIRES; Vogt et al. 1994) on Keck and the Magellan Inamori Kyocera Echelle instrument (MIKE; Bernstein et al. 2003) on the Magellan Clay telescope. NIR spectra were taken with the Gemini Near-Infrared Spectrograph (GNIRS) at Gemini North Observatory (Elias et al. 2006), the Folded-port InfraRed Echellette (FIRE; Simcoe et al. 2013) on Magellan Baade, SpeX (Rayner et al. 2003) on the NASA Infrared Telescope Facility (IRTF), and the Son OF ISAAC (SOFI) spectrograph mounted on the NTT (Moorwood et al. 1998).

\section{Reddening Estimation}

The Milky Way line-of-sight reddening for NGC 988 is $E(B-V)_{\mathrm{MW}}=0.024 \mathrm{mag}$ (Schlafly \& Finkbeiner 2011). Elias-Rosa et al. (2017) noted strong host $\mathrm{Na}$ I D absorption with an equivalent width (EW) of $1.45 \AA$ on day 6 , resulting in an estimation of a total $E(B-V)_{\text {tot }}=0.23$ using the relation presented in Turatto (2003). From the high-resolution Keck HIRES spectrum taken $\sim 6 \mathrm{hr}$ after discovery (Figure 2, top) we measure EWs of the individual Na I D lines of 0.75 and $0.62 \AA$ similar to the combined value found by Elias-Rosa et al. (2017). Unfortunately, the relationship between Na I D EW and dust extinction presented in Poznanski et al. (2012) saturates around $0.2 \AA$, requiring alternative methods for the reddening estimation of SN $2017 \mathrm{gmr}$.

From the same early high-resolution spectrum we also detect the $5780 \AA$ diffuse interstellar band absorption feature (Figure 2, bottom), which can be used to estimate the extinction $A_{V}$ (Phillips et al. 2013). We obtain an EW of $0.22 \AA$, which corresponds to $A_{V}=1.14 \mathrm{mag}, \quad$ or an $E(B-V)_{\mathrm{tot}}=0.36 \mathrm{mag}$ using an $R_{V}=3.1$ and the reddening law of CCM (Cardelli et al. 1989). Note that this relation was developed for $\mathrm{SNe}$ Ia and the uncertainty from this relationship is limited to $\pm 50 \%$, which only constrains the extinction to between $A_{V} \approx 0.6$ and $1.7 \mathrm{mag}$.

We also compare the $B-V$ color of SN 2017gmr during the plateau phase to other Type II SNe with published reddening estimates and adjust the $E(B-V)$ accordingly until we have a similar fit (similarly to that done by Tartaglia et al. 2018). Comparison with SN 2004et (Sahu et al. 2006), SN 2012A (Tomasella et al. 2013), SN 2013ab (Bose et al. 2015b), SN 2013ej (Bose et al. 2015a), and SN 2014cx (Huang et al. 2016), shown in Figure 3, constrains the reddening to $E(B-V)=$ $0.30 \pm 0.1 \mathrm{mag}$.

As another constraint we have compared our unreddened spectra with optical spectra of SN 2004et, a prototypical Type II$\mathrm{P}$, from similar epochs and applied reddening corrections until the spectra had a matching continuum slope. SN 2004et has a measured $E(B-V)=0.43 \mathrm{mag}$ (Sahu et al. 2006), and comparisons on both day 7 and day 84 yield a total $E(B-V)=0.30 \mathrm{mag}$ in SN 2017gmr. As this value is consistent with the other two estimates, we settle on a value of $E(B-V)=0.30 \mathrm{mag}$ as our final reddening estimation, with the caveat that there may be somewhat large uncertainties. This is the standard value that will be used throughout the paper.

\section{Photometric Evolution}

\subsection{Optical Light Curve}

The full optical light curve can be seen in Figure 4, and the $V$-band light curve compared to other Type II $\mathrm{SNe}$ is shown in Figure 5. For reference, the $r$-band discovery magnitude is
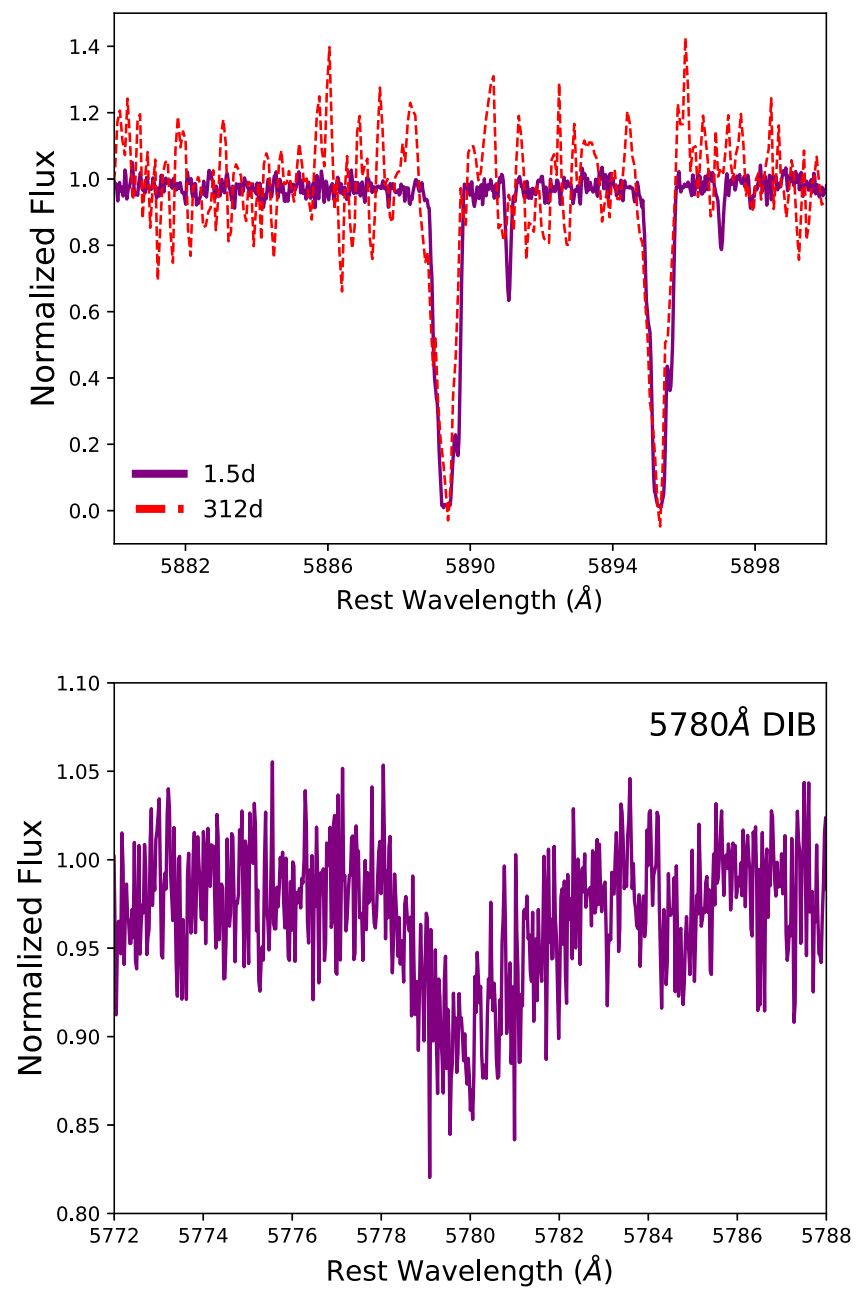

Figure 2. Keck HIRES spectra (purple) from day 1.5 showing the region around the $\mathrm{Na}$ I D lines (top) and the $\lambda 5780 \mathrm{DIB}$ feature (bottom). The red Na I D spectra is from Magellan/MIKE echelle spectra on day 312.

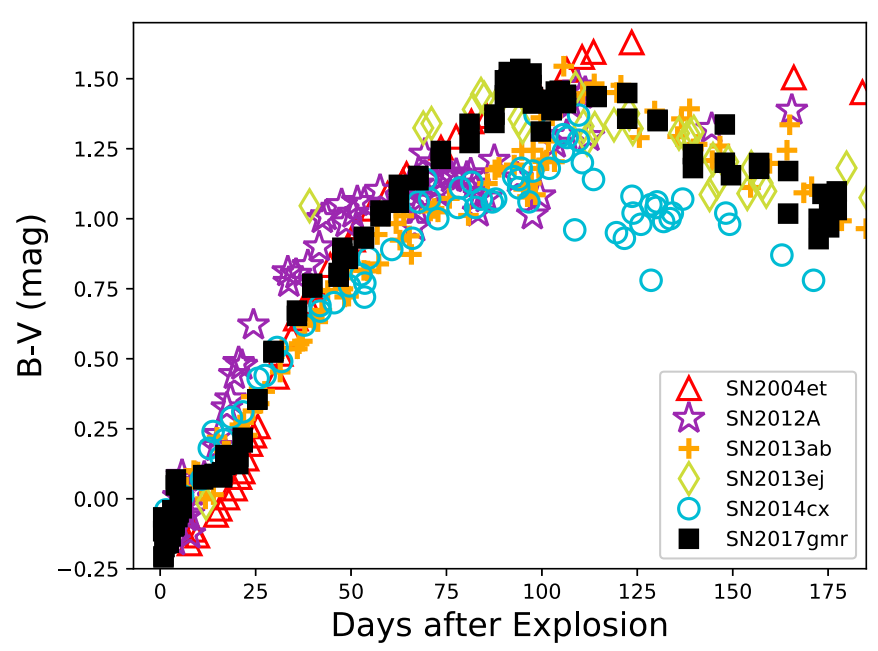

Figure 3. $B-V$ color evolution of SN 2017gmr (black) compared with other Type II-P SNe from the literature. All data have been corrected for reddening as indicated from the corresponding references. The data come from sources listed in Section 3.

shown as an open hexagon, while the dotted line connects the pre-explosion upper-limit $r$-band magnitude 2 days prior in Figure 5. Overall the shape is that of a typical Type II SN with 


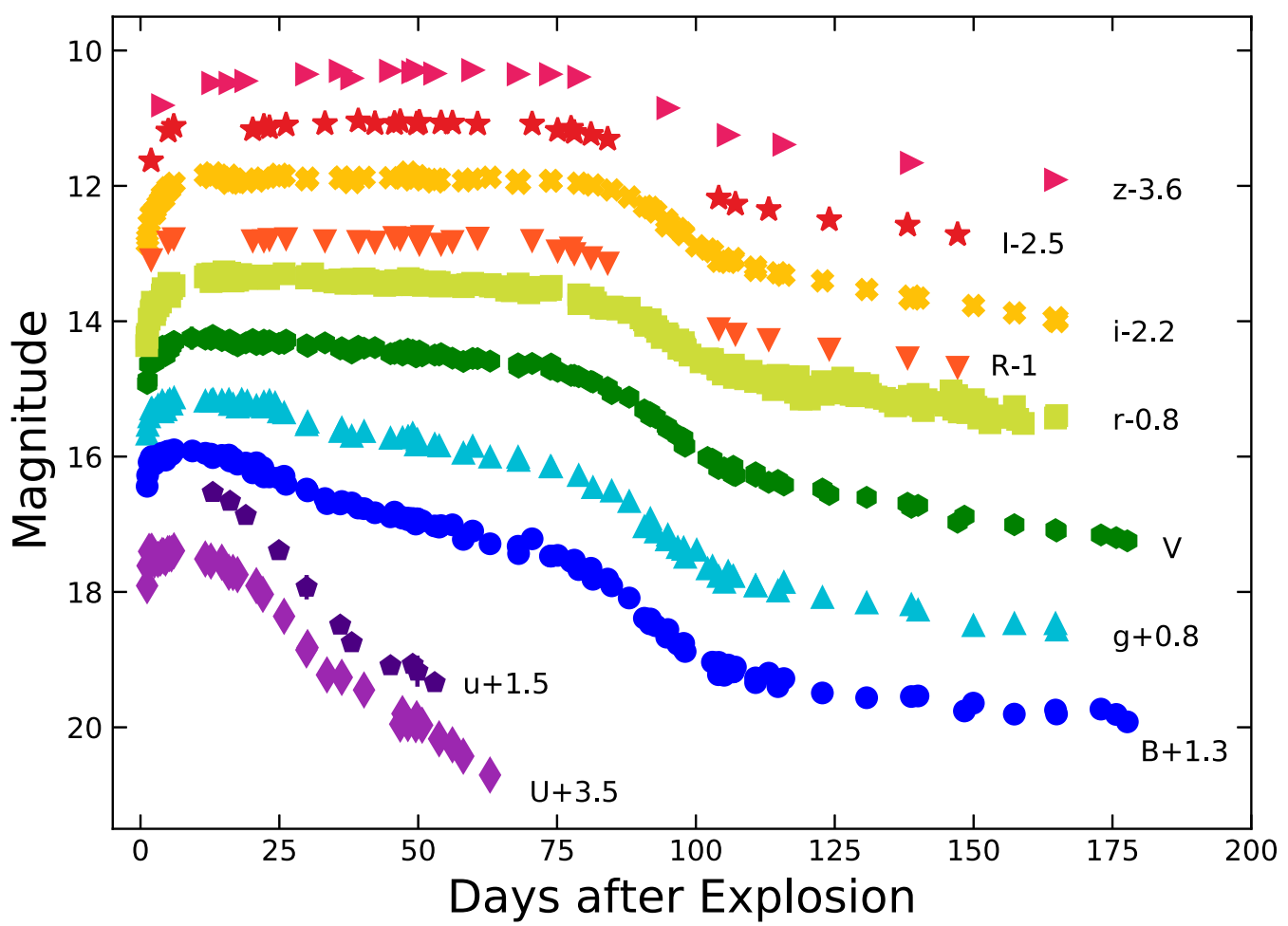

Figure 4. Optical photometry of SN $2017 \mathrm{gmr}$, shifted by constants for ease of viewing. Marker size is larger than uncertainties. The data set is tabulated in Table 1. The adopted date of explosion is considered to be MJD 57,999.09 (2017 September 3.1 UT) as described Section 4.8.

an extended plateau, albeit on the brighter end with a maximum $M_{V}=-18.3 \mathrm{mag}$. The maximum occurs at $\sim 6$ days after explosion for the $U$ and $B$ bands, $\sim 8$ days for $g$ and $V$, and closer to 10 days for $r$ and $i$. This is consistent with the average rise times seen for the majority of Type II SNe (GonzálezGaitán et al. 2015; Rubin et al. 2016; Förster et al. 2018).

The light curves then remain at a relatively constant magnitude for the next 75 days until the fall off the plateau begins around day 85 , with decline rates of $0.027,0.011$, and $0.003 \mathrm{mag} \mathrm{day}^{-1}$ in $B, V$, and $i$, respectively. Using the method described in Valenti et al. (2016), we obtain the point at half of the fall at MJD 58,093.5 \pm 0.4 , or 95 days after our estimated explosion date. Between day 85 and day 105 the $V$-band light curve drops by $1.5 \mathrm{mag}$. This moderate post-plateau drop is on the lower end but consistent with other Type II-P SNe, particularly higher-luminosity events (Valenti et al. 2014).

The plateau length of SN 2017gmr is on the shorter side for comparable objects and has an average $M_{V}=-17.8 \mathrm{mag}$ (Figure 5), a value noticeably brighter than the norm (but similar to SN 2004et). According to Anderson et al. (2014), Faran et al. (2014), and Galbany et al. (2016), more luminous Type II-P SNe tend to exhibit shorter plateau durations, which coincides with the overall picture of SN 2017gmr. SN 2017gmr, SN 2013fs, SN 2004et, and SN 2008if all show similar luminosities and evolution over the first few days (Figure 5 inset) but then evolve to drastically different light-curve shapes. While SN 2017gmr and SN 2004et change very little over the first 3 months, SN 2013fs and SN 2008if show evolution more akin to Type II-L SNe, with a larger drop in luminosity over the first $\sim 75$ days.

\subsection{The Early U-bump}

One rather intriguing feature seen in the early light curve of SN 2017gmr is the bump in luminosity that occurs a couple of days post-explosion, particularly in the bluest bands. In Figure 6 we show the ground-based $U$ and $B$ observations along with the Swift UV. From the $U$ and $B$ data we see a sharp rise over the first 2 days, then a drop of roughly 0.2 and $0.1 \mathrm{mag}$ in $U$ and $B$, respectively, and then a slow rise over the next few days back to the peak value. Unfortunately, no Swift data exist prior to day 2, so the light-curve behavior in the UV bands is unknown over the same time period. It is also possible that we are seeing undulations in the $U$ and $B$ light curves due to inhomogeneities in the CSM, particularly in some cases where the magnitude changes are larger than the uncertainties.

Models recently produced by Moriya et al. (2018) do show this small bump in luminosity in the $u$ and $g$ bands with certain mass-loss and density configurations (see also Morozova et al. 2018). The key to creating this early bump is to have moderately dense CSM close to the progenitor. The Type IIP SN 2016X showed a similar bump in the Swift $U V$ light curve over the first few days after explosion, although it did not seem to be present in the optical bands (Huang et al. 2018). Their explanation for the initial light-curve peak was a shock breakout cooling effect, but as we discuss in Section 4.8, we cannot fit this bump with standard shock-cooling models.

\subsection{Late-time Light Curve}

As we show in Figure 5, the radioactive tail of SN 2017gmr does not show the exponential decline of ${ }^{56} \mathrm{Co}$ decay of 0.98 mag 100 day $^{-1}$ (Woosley et al. 1989). While the $B$ band declines around 0.9 mag 100 day $^{-1}, V$ and $i$ decline by 1.5 and $1.4 \mathrm{mag} 100 \mathrm{day}^{-1}$, respectively. These values are more consistent with the mean value of $1.47 \mathrm{mag} 100 \mathrm{day}^{-1}$ in the $V$ band found by Anderson et al. (2014). By our last photometric observations around day 175 , the $V$-band light curve is about $0.5 \mathrm{mag}$ fainter than expected if we assume the 


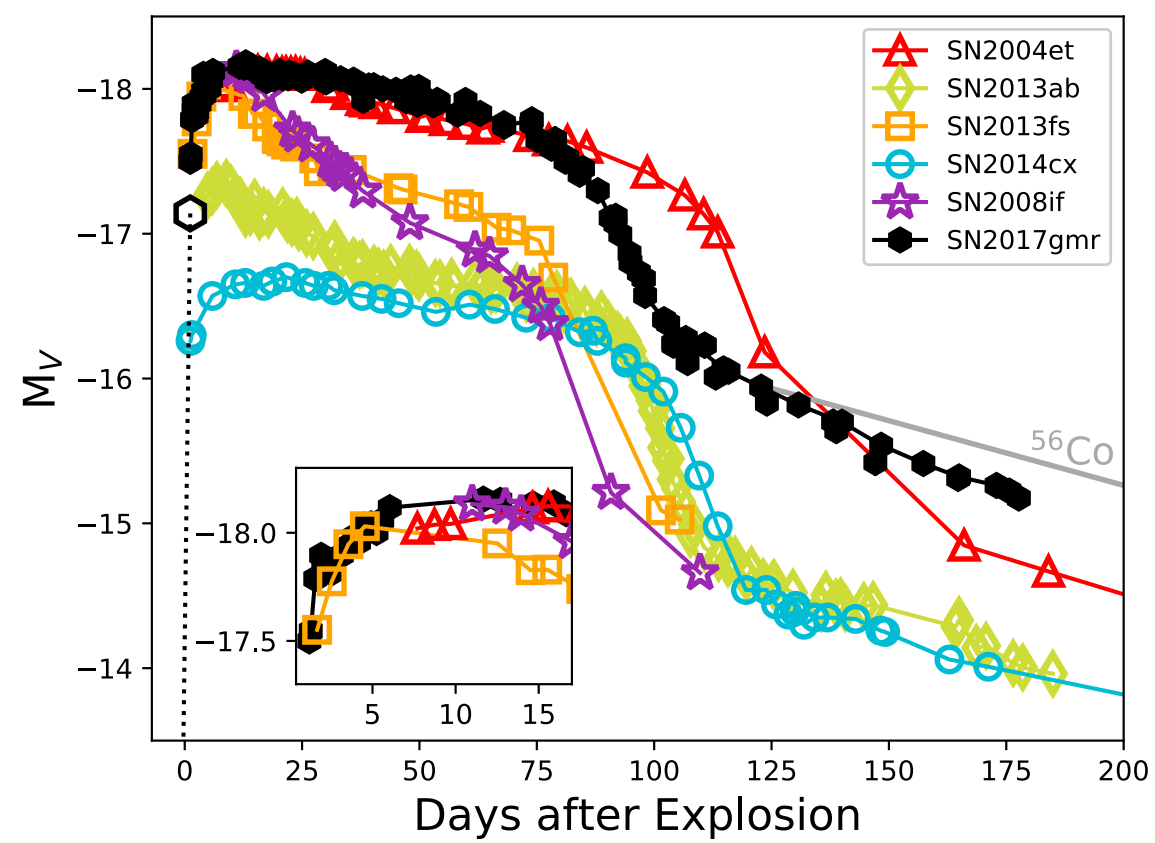

Figure 5. Absolute $V$-band light curves of a sample of Type II SNe. The inset in the lower left shows the comparison over the first 15 days among SN $2017 \mathrm{gmr}$, SN 2004et, SN 2013fs, and SN 2008if. Data are from Sahu et al. (2006; SN 2004et), Bose et al. (2015a; SN 20013ab), Huang et al. (2016; SN 2014cx), Gutiérrez et al. (2017a; SN 2008if), and Valenti et al. (2016; SN 2013fs).

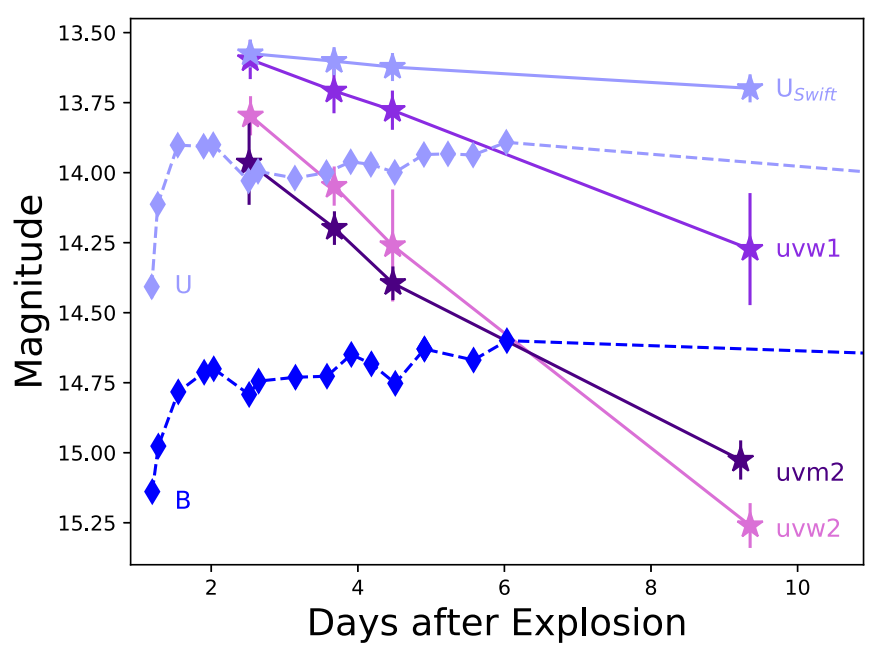

Figure 6. Swift photometry of SN $2017 \mathrm{gmr}$ compared with ground-based $U$ - and $B$-band photometry from Las Cumbres Observatory. The Swift photometry is tabulated in Table 2 .

canonical $0.98 \mathrm{mag} 100 \mathrm{day}^{-1}$. The same behavior is seen in the bolometric light curve, as we discuss below. This deviation can be explained by incomplete gamma-ray trapping, a decrease in the energy input from shock interaction, as dust production in the ejecta, or some combination of the three.

Incomplete gamma-ray trapping has been documented in other Type II-P SNe. As mentioned above, Anderson et al. (2014) found that the mean decline rates at late times for a large sample of Type II-P SNe were roughly $0.5 \mathrm{mag} 100$ day $^{-1}$ larger in $V$ band than the generally accepted value of $0.98 \mathrm{mag}$ $100 \mathrm{day}^{-1}$. They also found that the more luminous the SN, the greater the deviation from the expected decay rate. Highly energetic explosions can also have large expansion velocities, which in turn leads to weaker trapping. Alternatively, if the distribution of ${ }^{56} \mathrm{Ni}$ is very asymmetric or mixed in the ejecta, the escape probability could be greater. If CSM interaction is occurring, it can also contribute to the luminosity at late times and would not follow the predicted rate of ${ }^{56} \mathrm{Co}$ decay. We will discuss these possible scenarios further in Section 6.

\subsection{Infrared Light Curve}

Multiple epochs of NIR data were obtained over the first 160 days of evolution. The NIR luminosity rose over the first 30-40 days after explosion (Figure 7). This was followed by a few weeks of nearly constant luminosity; then, starting around day 75 , a steady decline begins in all filters and continues until our last observed epoch. We have plotted the NIR light curves of SN 2004et from Maguire et al. (2010) as a comparison, and they indicate that the NIR plateau is much shorter for SN 2017gmr than SN 2004et and that likely the late-time NIR luminosity is greater for SN $2017 \mathrm{gmr}$ as well.

\subsection{Color Evolution}

The $B-V$ color evolution of SN $2017 \mathrm{gmr}$ and a comparison to other $\mathrm{SNe}$ are shown in Figure 3. As in other Type II $\mathrm{SNe}$, the color is initially blue and evolves rapidly toward the red as the large envelope of the RSG progenitor expands and cools, until it reaches the recombination phase and the rate slows (de Jaeger et al. 2018a). This continues over the duration of the optically thick plateau phase until a peak value of $B-V=1.5$ mag. After day 100 , once the exponential decline phase begins, the color gradually becomes bluer again.

\subsection{Bolometric Light Curve}

The abundance of photometric data has allowed us to straightforwardly create a quasi-bolometric light curve using the routine SUPERBOL (Nicholl 2018). Following the description in Nicholl et al. (2016), the reddening and redshiftcorrected photometry in each band were interpolated with the $g$ 


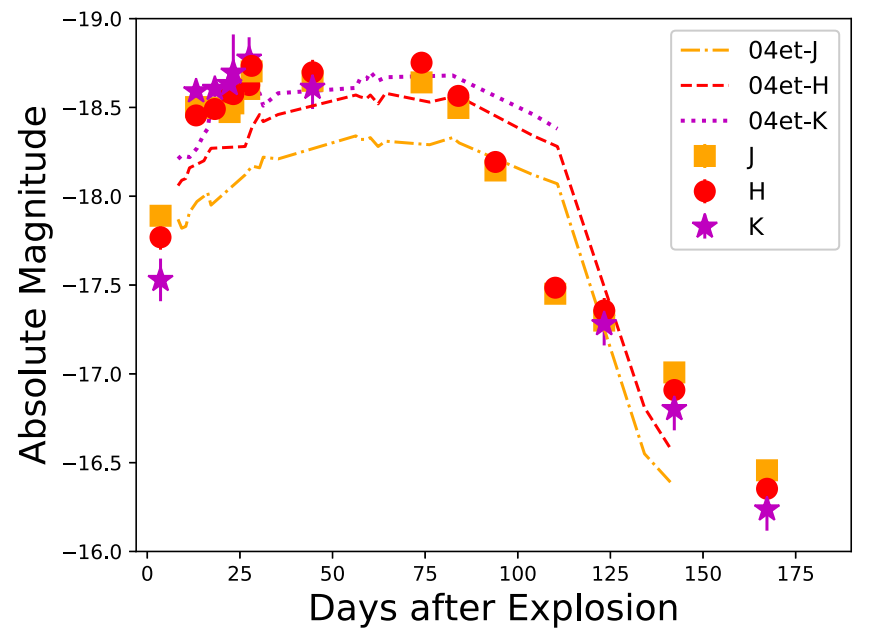

Figure 7. NIR light curve of SN $2017 \mathrm{gmr}$ in absolute magnitudes using $E(B-V)=0.30$ and $\mu=31.46 \mathrm{mag}$. Also shown for comparison is the NIR photometry of SN 2004et from Maguire et al. (2010) corrected for an $E(B-V)=0.41$ and a $\mu=29.4$ mag (Anand et al. 2018).

band as reference and then converted to a spectral luminosity $\left(L_{\lambda}\right)$. The bolometric luminosity was then computed from the integration of the SED for each epoch.

In Figure 8 we show the bolometric light curve produced from the observations (red) and those obtained with blackbody corrections (black), as well as the blackbody temperature $\left(T_{\mathrm{bb}}\right)$ and blackbody radius $\left(R_{\mathrm{bb}}\right)$ shown in orange and purple, respectively, in the bottom of Figure 8 . The red light curve is pseudo-bolometric and is constructed by integrating under the filters from UV to IR. Swift UV coverage does not extend past $\sim 9$ days, so a first-order polynomial is fit to the data and extended out to later epochs. As the contribution to the total bolometric luminosity falls quickly after the first few weeks, this does not add much uncertainty. The data have been corrected for an $E(B-V)=0.30 \mathrm{mag}$ and adopting the distance modulus $\mu=31.46 \mathrm{mag}$.

As we mention above, the late-time light curve falls faster than expected for a fully trapped ${ }^{56} \mathrm{Co}$ decay of $0.98 \mathrm{mag}$ 100 day $^{-1}$, with $L_{\text {bol }}$ roughly $1 \times 10^{41} \mathrm{erg} \mathrm{s}^{-1}$ fainter that predicted on day 165 . Integrating over the entire bolometric light curve gives a total radiated energy of $3.5 \times 10^{49} \mathrm{erg}$ in the first 175 days.

\subsection{A Search for Pre-SN Outbursts}

With the advent of high-cadence transient searches in the past decade, several instances of pre-SN outbursts have been observed directly in the months to years before explosion (e.g., Fraser et al. 2013; Mauerhan et al. 2013; Ofek et al. 2013, 2014; Elias-Rosa et al. 2016; Tartaglia et al. 2016; Reguitti et al. 2019), although overall detectable outbursts are rare (Bilinski et al. 2015; Strotjohann et al. 2015). These outbursts are generally associated with $\mathrm{SNe}$ that have substantial circumstellar material as evidenced by their SN IIn-like behavior. However, many standard Type II-P/L SNe also show evidence for CSM material either as narrow emission lines in their early-time spectra (e.g., Khazov et al. 2016) or as early peaks in their light curves (Morozova et al. 2017). This CSM could have been deposited in the years or decades prior to explosion and could have been accompanied by faint pre-SN outbursts, as has recently been suggested in the gravity-wavedriven scenario of Shiode \& Quataert (2014) and Fuller (2017),
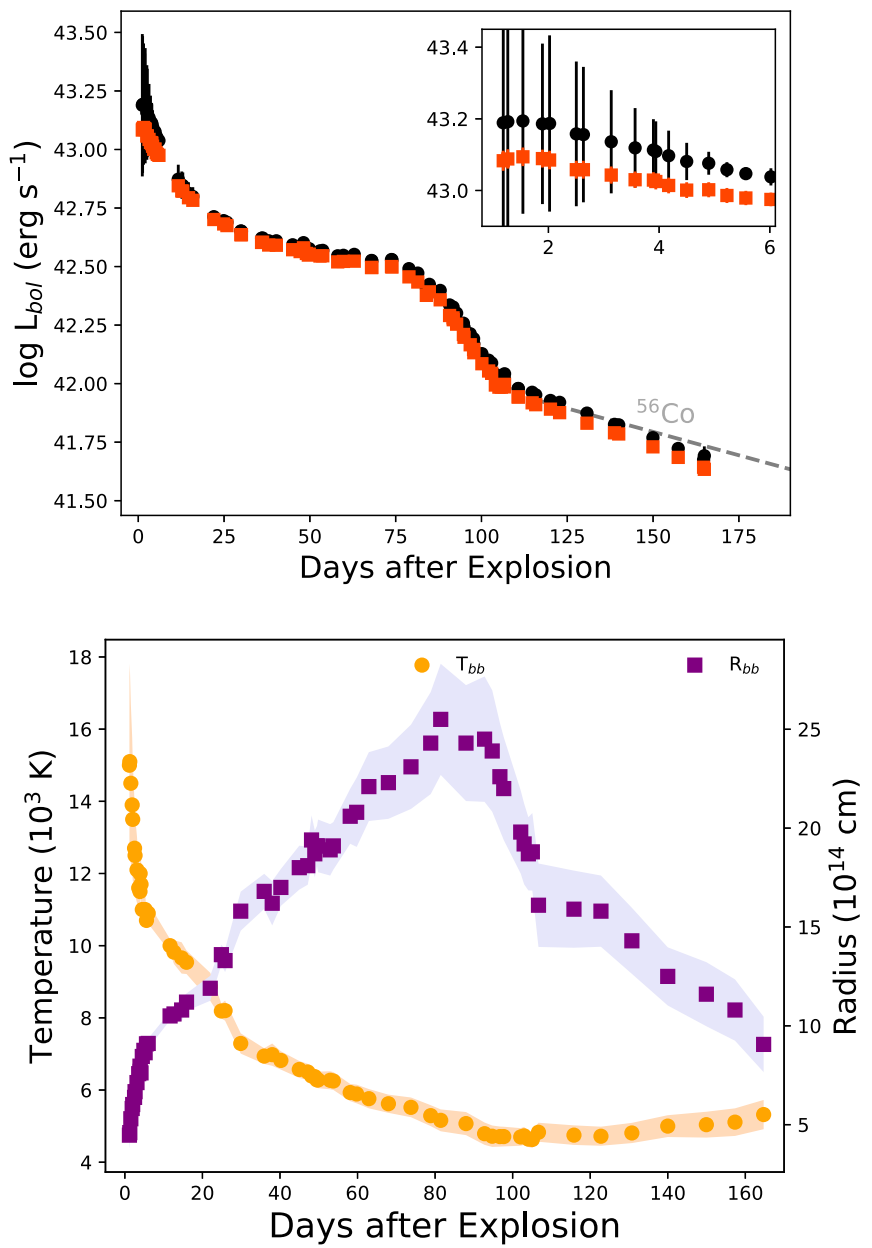

Figure 8. Top: bolometric light curve integrated from near-UV to NIR. The inset shows a zoom-in of the first few days. The red squares indicate the observed luminosity, while the black circles come from blackbody corrections to the data. The ${ }^{56} \mathrm{Co}$ decay rate is indicated in gray. Bottom: blackbody temperature and radius evolution of SN $2017 \mathrm{gmr}$ derived from the photometry. The temperature is plotted in orange, and the radius is plotted in purple. The uncertainties are indicated by the shaded regions.

or in unsteady nuclear burning events or binary interaction (Smith \& Arnett 2014).

The field of NGC 988 was observed by the DLT40 survey 56 times between 2015 January and 2017 September, just prior to the explosion of SN 2017gmr. During much of this time period, the DLT40 survey was coming online, with some prolonged down periods. No precursor outbursts were observed down to a typical limiting magnitude of $r \sim 19-19.5 \mathrm{mag}$ $\left(-12>M_{r}>-12.5\right)$. We can therefore rule out bright eruptions like SN imposters or LBV eruptions with roughly $M_{r}=-14$ mag lasting several months, but not fainter or shortlived outbursts. This includes those LBV eruptions that have been found to have magnitudes of only $M_{r}=-10$ or $-11 \mathrm{mag}$ (Smith et al. 2011b).

\subsection{Early Light-curve Modeling}

Due to the well-sampled photometric data over the first few days after explosion in SN 2017gmr, we are able to model the early-time light curves using the prescriptions outlined in Sapir \& Waxman (2017). To do this, we employed the code presented in Hosseinzadeh (2019) and described in Hosseinzadeh et al. (2018), which uses a Markov chain Monte Carlo (MCMC) 
routine to fit the light curve in each photometric band and outputs posterior probability distributions for physical parameters, such as the time of explosion, the temperature and luminosity 1 day after explosion, the time at which the envelope becomes transparent, and the progenitor radius. Data were only fit up to day 4 to still lie within the validity range described by Rubin \& Gal-Yam (2017). The best fits to our data are shown in Figure 9.

One day after explosion, the modeled temperature is $(25.9 \pm$ $0.1) \times 10^{3} \mathrm{~K}(\mathrm{kK})$ with a luminosity of $(2.9 \pm 0.03) \times$ $10^{43} \mathrm{erg} \mathrm{s}^{-1}$. From the Sapir \& Waxman (2017) fits the estimated progenitor radius is $489 \pm 22 R_{\odot}\left(3.4 \times 10^{13} \mathrm{~cm}\right)$, a value on the small end for an RSG, which theoretically can range in size from $\sim 100$ to $1500 R_{\odot}$ (Levesque 2017), but that is commensurate with observations of some Galactic RSGs (e.g., Wittkowski et al. 2017; Montargès et al. 2018). From these fits we also derive an explosion date of MJD 57,999.09 \pm 0.01 days. This value is further bolstered by our first observation obtained on MJD 58,000.27, or just over a day after the estimated explosion date, and our last nondetection on MJD 57,998.22. This is also consistent with the UV photometry obtained 2.5 days after discovery, which does not show a rise to peak that is seen in other bands (Figure 6).

\section{Spectroscopic Evolution}

\subsection{Optical Spectra}

The early spectra, shown in Figure 10, are typical for a young Type II-P SN, displaying a blue, mostly featureless continuum. Only strong interstellar $\mathrm{Na}$ I D absorption lines and a broad emission feature around $4600 \AA$ (likely He II $\lambda 4686$ ) are seen. Neither the low-resolution FLOYDS spectrum nor the high-resolution Keck spectrum, taken within hours of discovery, shows signs of narrow high-ionization lines, other than narrow $55 \mathrm{~km} \mathrm{~s}^{-1} \mathrm{H} \alpha$ seen in the Keck HIRES echelle spectrum. This is different from other early-detected CCSNe which can show features of highly ionized nitrogen and carbon along with He and $\mathrm{H}$. This is discussed further in Section 6.3.

As the photosphere begins to cool, the continuum becomes redder and broad Balmer emission lines begin to appear with $\mathrm{P}$ Cygni absorption features. When $\mathrm{H} \alpha$ becomes pronounced a week after explosion, the peak appears blueshifted, centered at $-5000 \mathrm{~km} \mathrm{~s}^{-1}$. This is a common occurrence in Type II-P SNe, where the opaque hydrogen envelope preferentially obscures the redshifted, receding side of the line (Dessart \& Hillier 2005a; Anderson et al. 2014). As the recombination front moves through the envelope, the red side becomes visible again and the emission-line peak becomes more symmetric.

Around a month after explosion, the $\mathrm{SN}$ is well into the plateau phase and the Ca II IR triplet centered around $8600 \AA$ emerges, along with a forest of metal lines blueward of $5000 \AA$ (Figure 11). In particular, lines of Fe II, including Fe II $\lambda \lambda 4924$, 5018, and 5169, can be seen.

By the end of the plateau phase other broad lines such as Ba II $\lambda 6142$, [Sc II] $\lambda \lambda 5527,5658$, and 6246 (blended with [O I]), and [O I] $\lambda \lambda 6300,6364$ appear in the nebular spectra (Figure 12). Redward of $\mathrm{H} \alpha$, strong [Ca II] $\lambda \lambda 7291,7324$ is seen, flanked on either side by He I $\lambda 7065$, Fe II $\lambda 7155$, and O I $\lambda 7774$. What appears to be $\mathrm{K} \mathrm{I} \lambda \lambda 7665,7699$ is also detectable and distinct from O I by $\sim$ day 120 . The emergence of the He I $\lambda 7065$ line starting around day 90 suggests the presence of a strong ionization source. Also of note is the strengthening of the Ca II IR triplet, which has become almost as strong as $\mathrm{H} \alpha$ by day 165 .

\subsection{IR Spectra}

Figure 13 shows the NIR spectral evolution from 2 to 149 days. Overall the spectra show a decrease in flux with increase in wavelength, typical of young CCSNe. The spectra from the first week are featureless (minus atmospheric absorption), but by day 13 some $\mathrm{Pa} \alpha$ emission begins to emerge. Over the next month $\mathrm{Pa} \beta$ and $\mathrm{Br} \gamma$ appear as the continuum flux decreases. Both He I 1.083 and $\mathrm{Pa} \gamma$ are present, although slightly blended. As the SN drops from the plateau phase after 100 days, additional lines of O I, Si I, He I, and other weak hydrogen series are seen. The $\mathrm{CO}$ overtone between 2.3 and $2.5 \mu \mathrm{m}$ is not present in our last two spectra as has been seen for other Type II SNe (Yuan et al. 2016; Rho et al. 2018; Sarangi et al. 2018; Tinyanont et al. 2019). This may help rule out dust formation, at least in the first 150 days.

\subsection{Distance Measurements}

To help constrain the distance to SN 2017gmr, we have used the Expanding Photosphere Method (EPM; Kirshner \& Kwan 1974), which relies on the relation between the photometric angular radius and the spectroscopic physical radius of the homologously expanding SN ejecta. Assuming that the outflow is radiating as a diluted blackbody, the observed SN magnitudes are fitted to a blackbody function multiplied by dilution factors, to derive the color temperature and the angular radius. Dilution factors based on atmosphere modeling of Type II SNe were adopted from Dessart \& Hillier (2005b). Further, to eliminate the effect of filter response function ingrained in the observed broadband magnitudes, the response function is convolved with the blackbody model flux. The convolved function can be expressed in terms of the color temperature and the coefficient values taken from Hamuy et al. (2001). Following the same procedure undertaken in Dastidar et al. (2018), expansion velocities were calculated using the He I $\lambda 5876$ and Fe II $\lambda 5169$ lines over the first 50 days of evolution.

The distance is derived from a linear fit to the data in the form of

$$
t=D\left(\theta / v_{\mathrm{ph}}\right)+t_{o},
$$

where the slope is the distance and the $y$-intercept the date of explosion. This fit is shown in Figure 14. From this method we obtain an EPM distance of $18.6 \pm 2.2 \mathrm{Mpc}$, a value consistent with the $19.1 \mathrm{Mpc}$ used throughout the paper. It also indicates an explosion epoch of MJD 57,999.0 \pm 1.9 days, which agrees well with the constrained explosion date discussed above.

We have also measured the distance using the Standard Candle Method (SCM), which was first proposed by Hamuy \& Pinto (2002) and later expanded on by other authors. SCM uses photometric magnitudes and expansion velocities at 50 days. For SN $2017 \mathrm{gmr}$ these values are $m_{V}=14.57 \pm 0.04, m_{R}=$ $13.86 \pm 0.02, m_{I}=13.56 \pm 0.03$, and $v_{\mathrm{Fe}}$ II $=5600 \mathrm{~km} \mathrm{~s}^{-1}$. From these values we get SCM distances (in Mpc) of 16.10 (Hamuy 2005), 16.88 (Takáts \& Vinkó 2006), 24.70 (Nugent et al. 2006), 14.38 (Poznanski et al. 2009), 10.24 (de Jaeger et al. 2017), and 13.31 (Gall et al. 2018). Except for Nugent et al. (2006), all other SCM distances are systematically lower than the EPM and kinematic distances. The same was found for 


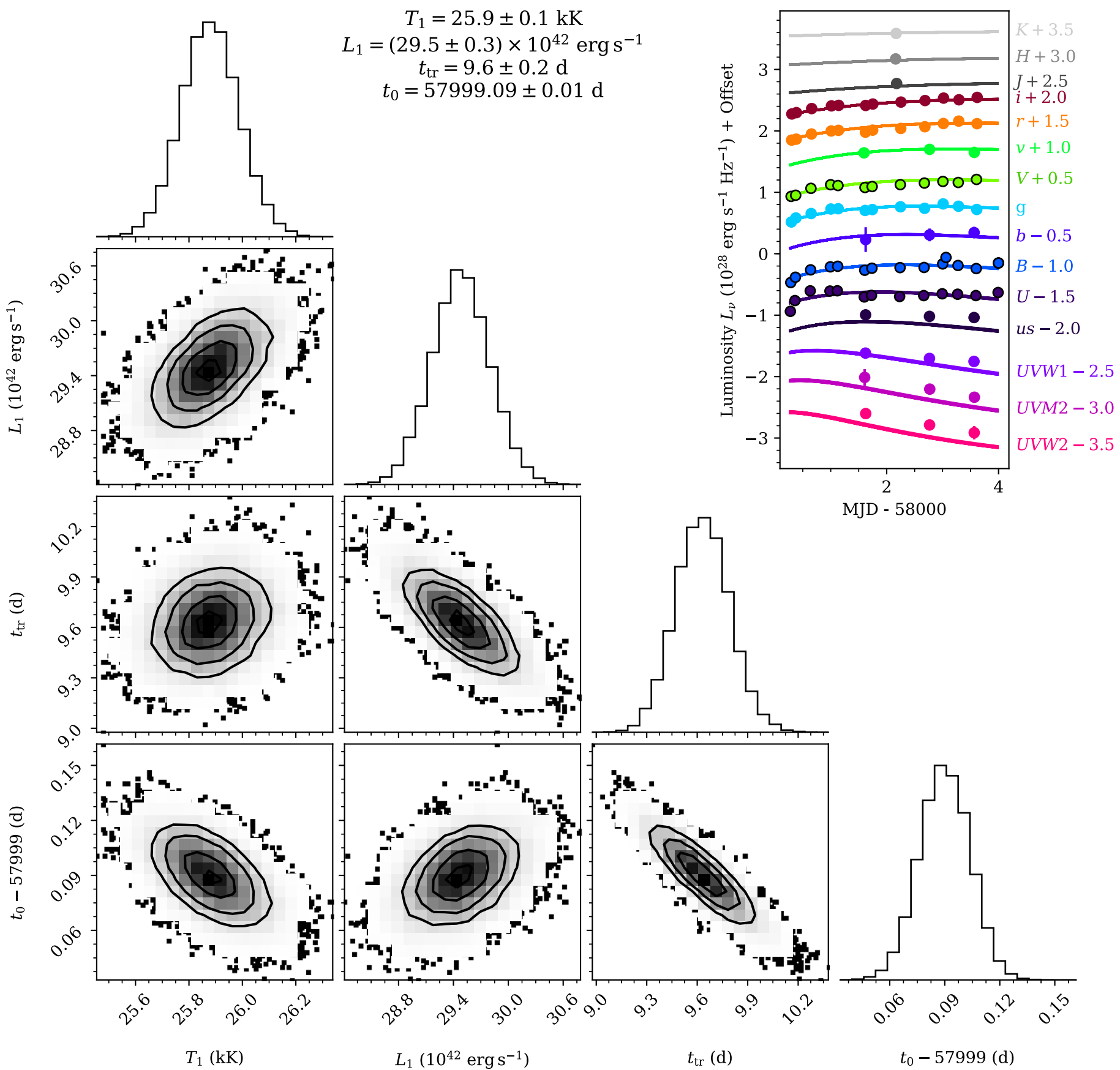

Figure 9. Posterior probability distributions of various parameters of SN $2017 \mathrm{gmr}$ calculated using the methods described in Hosseinzadeh et al. (2018), who applied them to the SN 2016bkv. We show the temperature and luminosity 1 day after explosion $\left(T_{1}, L_{1}\right)$, the time of explosion $\left(t_{0}\right)$, and the time to envelope transparency $\left(t_{\mathrm{tr}}\right)$. The top right panel shows 100 fits randomly drawn from the MCMC routine (Hosseinzadeh 2019) fit to the photometry. The fits appear as a thick solid line owing to the goodness of fit. Deviations between the light-curve points and the fits are likely due to early CSM interaction.

SN 2017eaw and SN 2004et in Szalai et al. (2019) and could be due to CSM interaction or asymmetries. The SCM method relies on a correlation between the magnitude and expansion velocity at day 50, which could break down under these conditions.

\section{Discussion \\ 6.1. ${ }^{56} \mathrm{Ni}$ Mass}

To estimate the ${ }^{56} \mathrm{Ni}$ mass, we employ various methods from the literature, in particular those of Hamuy (2003), Jerkstrand et al. (2012), and Pejcha \& Prieto (2015). These methods all rely on bolometric luminosities in the radioactive tail phase, so we use the constructed bolometric light curve discussed above (Figure 8). This results in measured ${ }^{56} \mathrm{Ni}$ masses of $0.130 \pm 0.026 M_{\odot}$, $0.124 \pm 0.026 M_{\odot}$, and $0.090 \pm 0.030 M_{\odot}$, respectively, for the three techniques. In the Pejcha \& Prieto (2015) calculation, we extrapolated the bolometric luminosity to day 200 and obtain an $L_{\text {bol }}=(1.85 \pm 0.9) \times 10^{41} \mathrm{erg} \mathrm{s}^{-1}$.

Other than SN $1992 \mathrm{H}$, for which the actual ${ }^{56} \mathrm{Ni}$ mass could be as low as $0.06 M_{\odot}$ depending on the distance used, and SN 1992am (Hamuy 2003), this is one of the highest ${ }^{56} \mathrm{Ni}$ masses reported for normal Type II SNe (Anderson 2019), higher if there is incomplete gamma photon trapping or if the $\mathrm{SN}$ is at a further distance than $19.6 \mathrm{Mpc}$, and lower if there is 


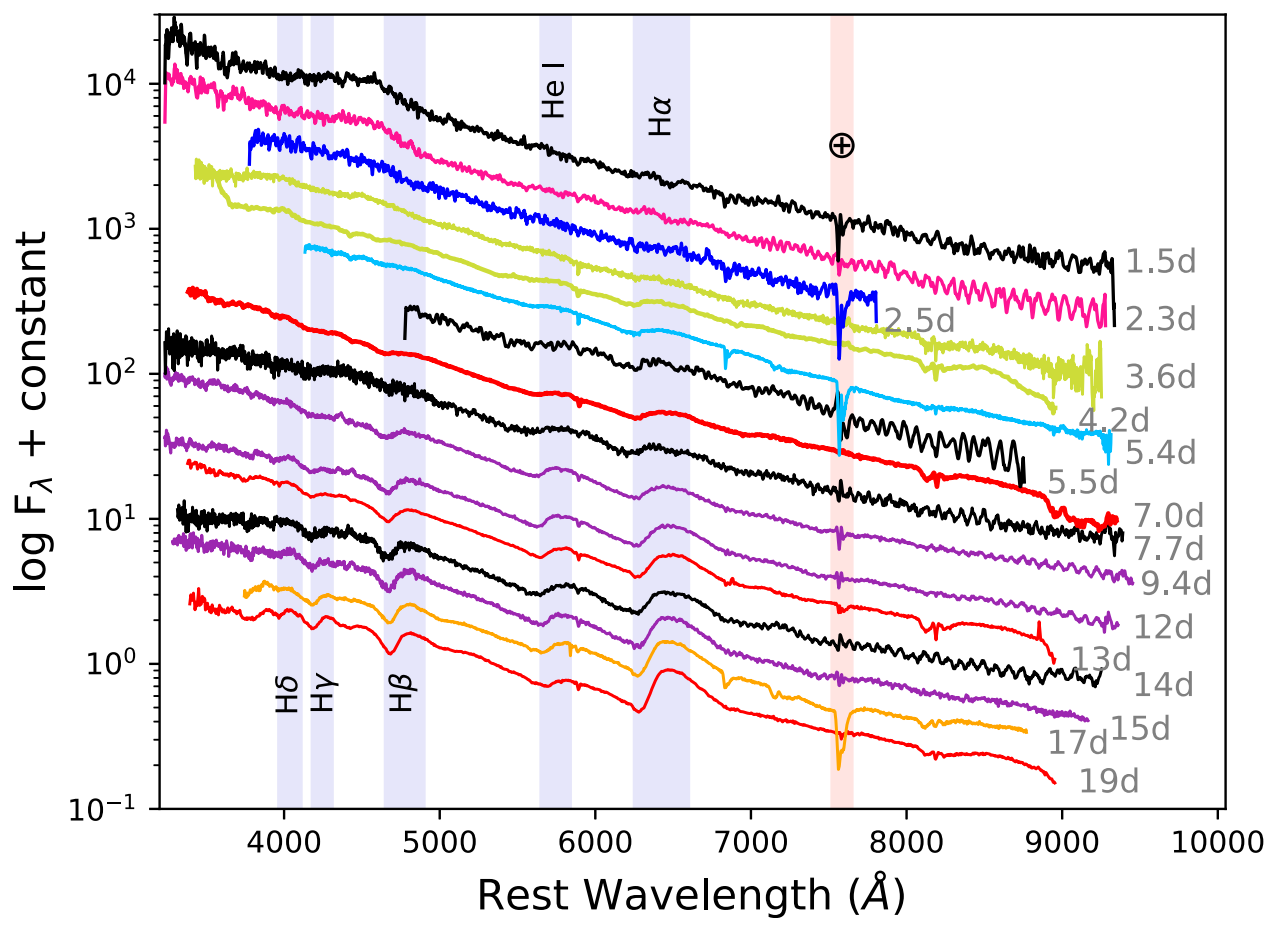

Figure 10. Optical spectral sequence of SN $2017 \mathrm{gmr}$ up until 19 days after explosion. The color of each spectrum represents a particular instrument+telescope pair that corresponds to the same post-explosion date as listed in the optical spectroscopy log presented in Table 4.

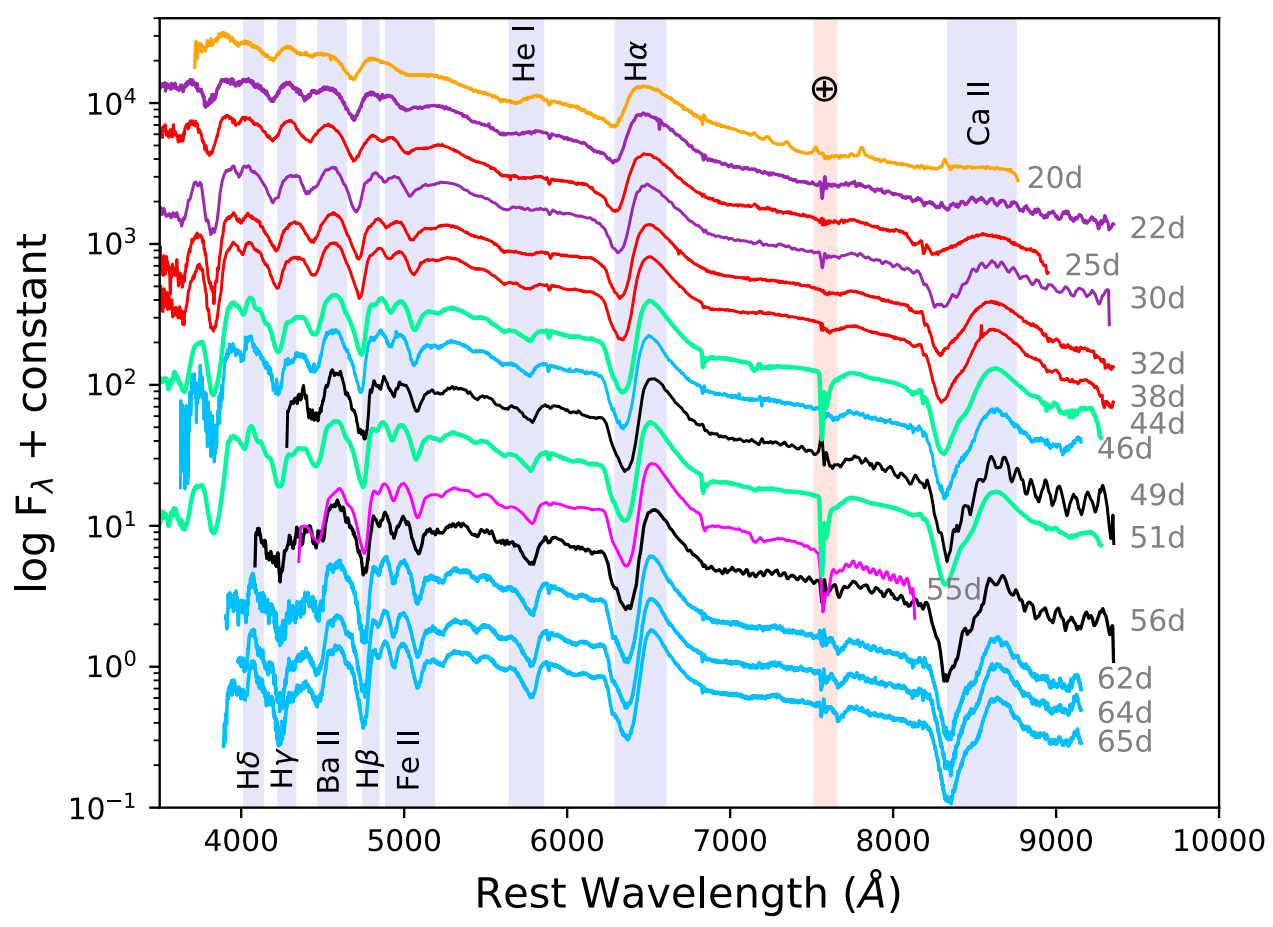

Figure 11. Same as Figure 10, but for 20-65 days after explosion.

CSM interaction or if the $\mathrm{SN}$ is closer. According to Müller et al. (2017), less than $5 \%$ of Type II-P SNe have ${ }^{56} \mathrm{Ni}$ masses as large as $0.12 M_{\odot}$. For comparison, other "normal" Type II-P SNe such as SN 1999em, SN 2003gd, and SN 2004dj each have ${ }^{56} \mathrm{Ni}$ masses of $\sim 0.02 M_{\odot}$, or a full order of magnitude lower than estimated here (Elmhamdi et al. 2003b; Hendry et al. 2005; Vinkó et al. 2006).
We can also estimate the ${ }^{56} \mathrm{Ni}$ mass using a steepness factor $S$, where $S=-d M / d t$, a measure of the transition between the plateau and radioactive tail phases (Elmhamdi et al. 2003a). Generally an anticorrelation exists, where the steeper the transition, the lower the ${ }^{56} \mathrm{Ni}$ mass. Following Equation (7) in Singh et al. (2018), we measure a steepness factor $S=$ $0.070 \pm 0.007 \mathrm{mag} \mathrm{day}^{-1}$, which corresponds to an estimated 


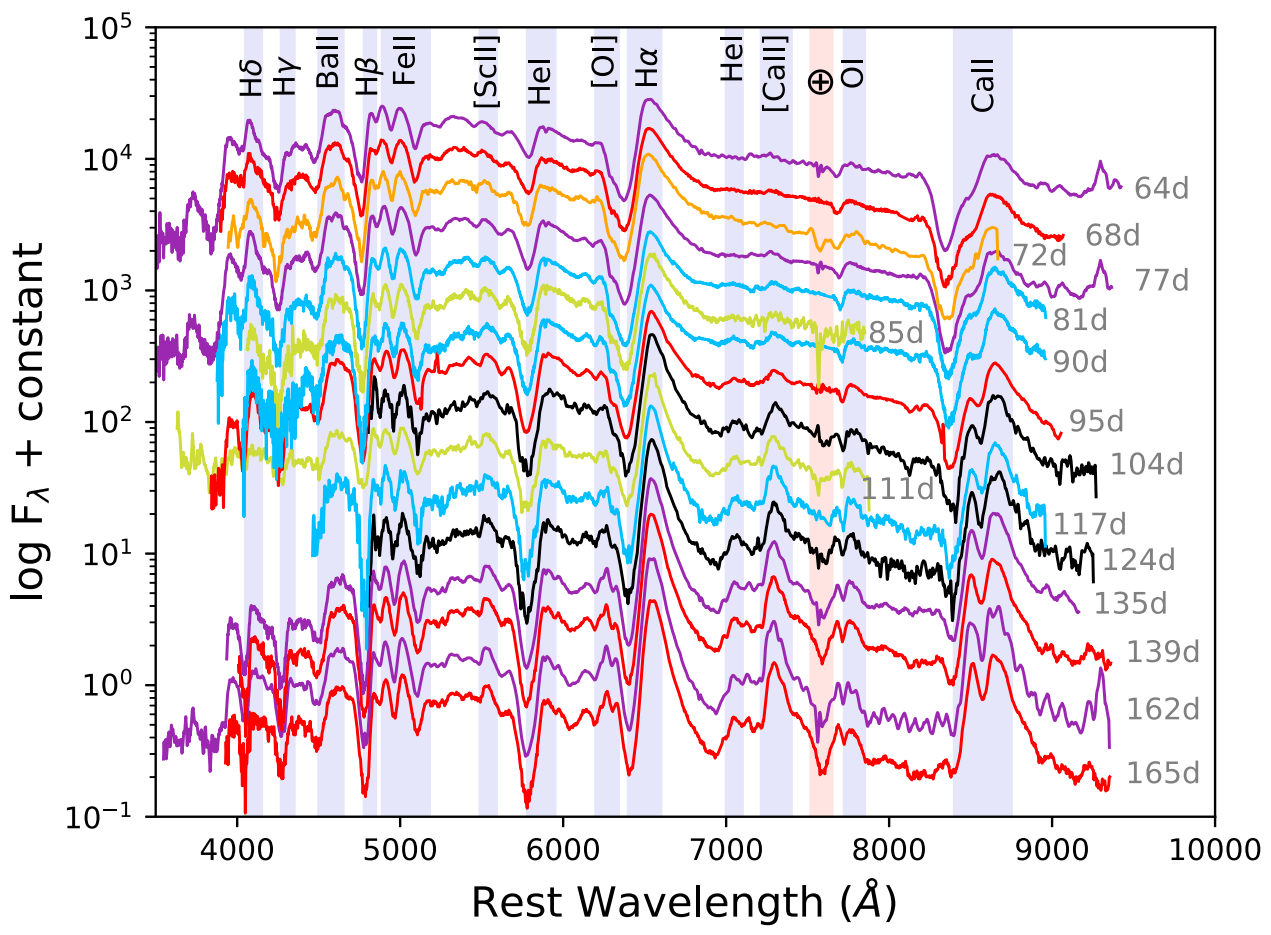

Figure 12. Same as Figure 10, but for 64-165 days after explosion.

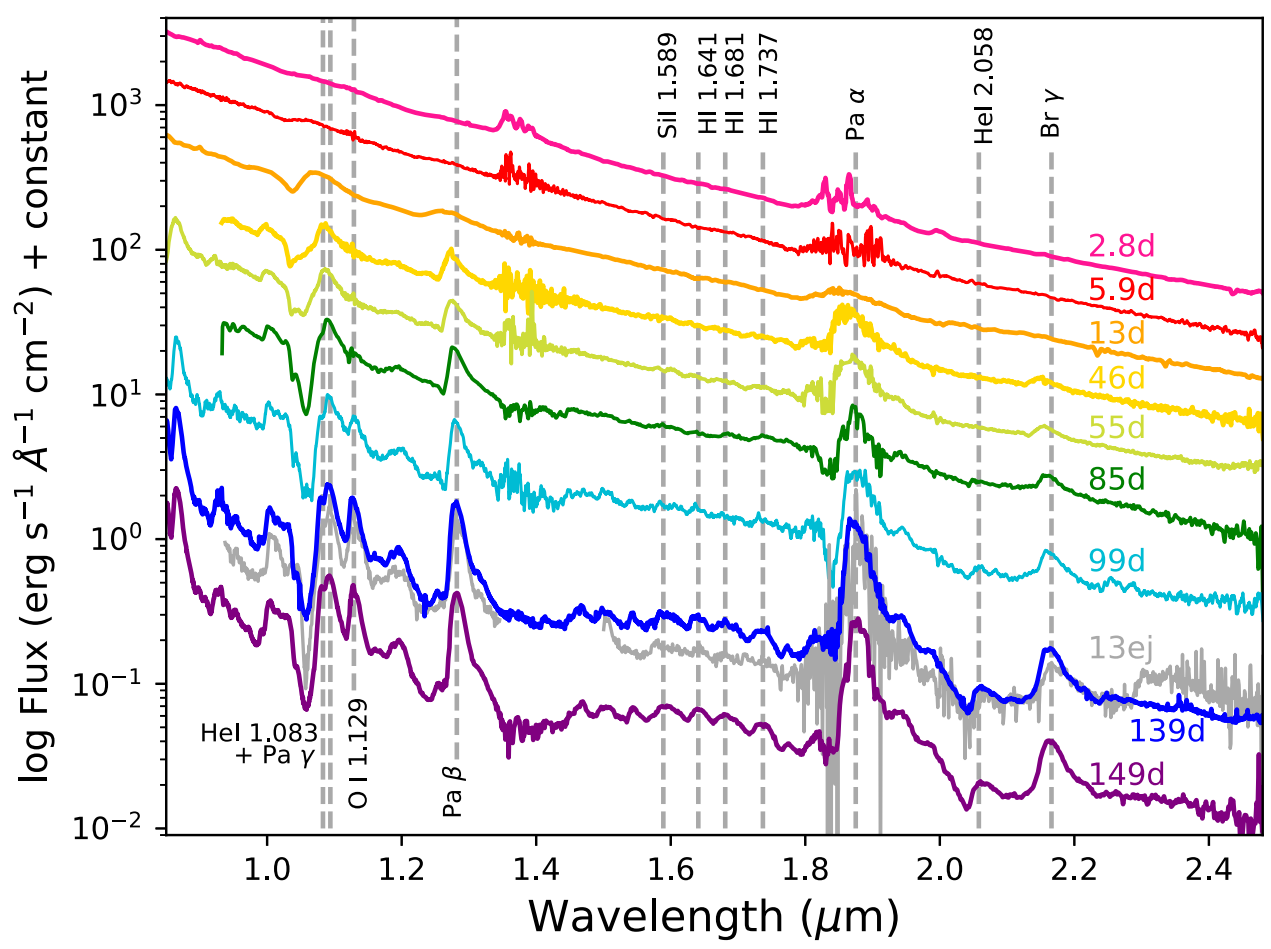

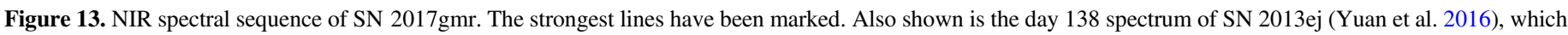
displays prominent $\mathrm{CO}$ overtone bands between 2.3 and $2.5 \mu \mathrm{m}$. An NIR spectroscopy log is presented in Table 5 .

${ }^{56} \mathrm{Ni}$ mass of $\sim 0.055 M_{\odot}$. This is significantly smaller than the value obtained using the late-time bolometric luminosity and more consistent with other normal Type II-P SNe. This inconsistency could be due to the degree of mixed ${ }^{56} \mathrm{Ni}$ in the ejecta, since the same amount of ${ }^{56} \mathrm{Ni}$ will create a steeper decline if it is centrally located rather than mixed. The mixed ${ }^{56} \mathrm{Ni}$ will actually increase the radiative diffusion timescale, causing the transition to appear shallower.

\subsection{Extremely Fast Ejecta}

In Figure 15 we show the evolution of the line velocities of both $\mathrm{H} \alpha$ and Fe II $\lambda 5169$ (shown as a function of radius over time). $\mathrm{H} \alpha$ falls from $15,000 \mathrm{~km} \mathrm{~s}^{-1}$ near explosion to a relatively stable value of $7000-8000 \mathrm{~km} \mathrm{~s}^{-1}$ during the radioactive tail. Fe II $\lambda 5169$, a more reliable measurement of photospheric velocity than $\mathrm{H} \alpha$, settles to a late-time velocity of 


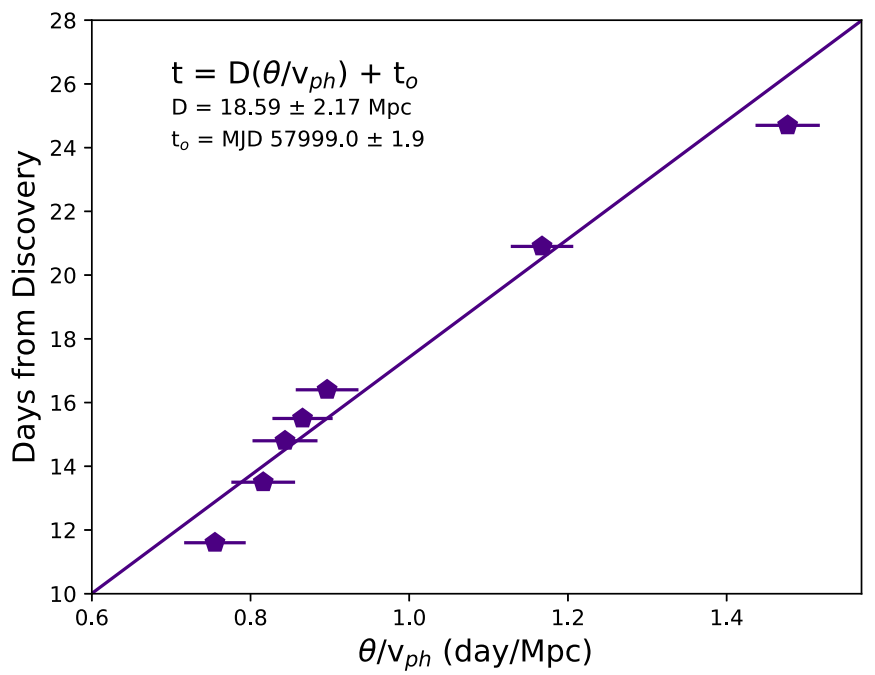

Figure 14. Distance determination using EPM for SN 2017gmr.

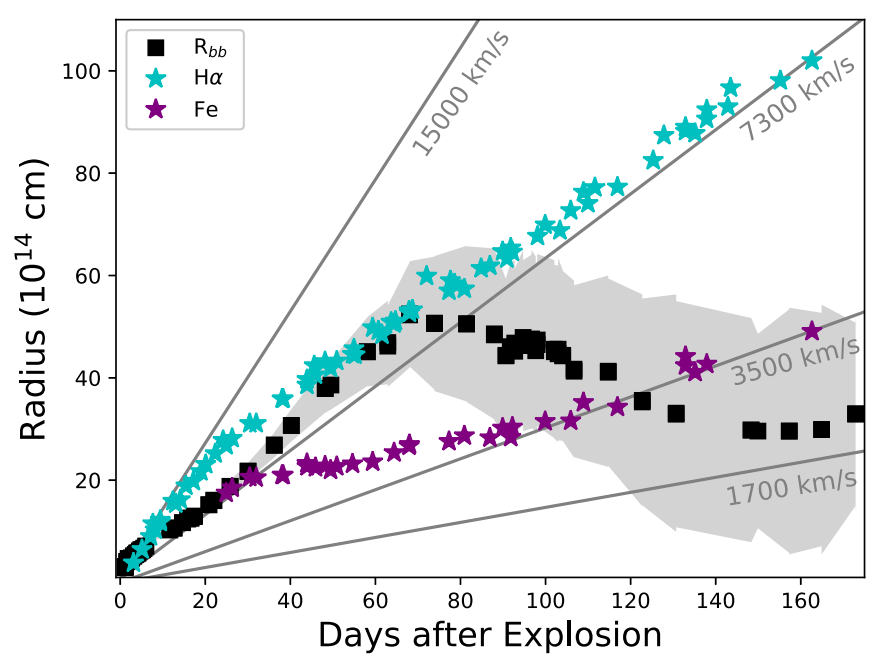

Figure 15. Blackbody radius of SN 2017gmr compared to the photospheric radius calculated from $\mathrm{H} \alpha$ and Fe II $\lambda 5169$. Lines of constant velocity are plotted in dark gray, while the uncertainty in $R_{\mathrm{bb}}$ is shown in light gray.

$3500 \mathrm{~km} \mathrm{~s}^{-1}$. These expansion velocities are higher than average for Type II SNe and for Type II-P SNe in particular. In Figure 16 we show the comparison of SN 2017gmr optical spectra at various epochs with the well-studied SN 1999em and SN 2004et. At all epochs the line velocities of SN 2017gmr are faster than those of the other two.

From Gutiérrez et al. (2017a), the mean velocities on day 53 for a sample of 122 Type II SNe (measured from the absorption minimum) are 6365 and $3537 \mathrm{~km} \mathrm{~s}^{-1}$ for $\mathrm{H} \alpha$ and Fe II $\lambda 5169$, respectively. In comparison, SN $2017 \mathrm{gmr}$ has velocities on day 53 of 9330 and $5240 \mathrm{~km} \mathrm{~s}^{-1}$ for $\mathrm{H} \alpha$ and Fe II 5169, respectively. By day 115, the difference in the Fe II $\lambda 5169$ velocities has decreased, $2451 \mathrm{~km} \mathrm{~s}^{-1}$ on average versus $3520 \mathrm{~km} \mathrm{~s}^{-1}$ for SN $2017 \mathrm{gmr}$, but $\mathrm{H} \alpha$ remains almost $2000 \mathrm{~km} \mathrm{~s}^{-1}$ faster than the mean value of $5805 \mathrm{~km} \mathrm{~s}^{-1}$.

The faster line velocities seem to correlate well with the high inferred ${ }^{56} \mathrm{Ni}$ mass and maximum luminosity of SN $2017 \mathrm{gmr}$. Gutiérrez et al. (2017b) found a correlation between expansion velocities and ${ }^{56} \mathrm{Ni}$ mass that indicated that more energetic explosions (resulting in faster expansion velocities) created

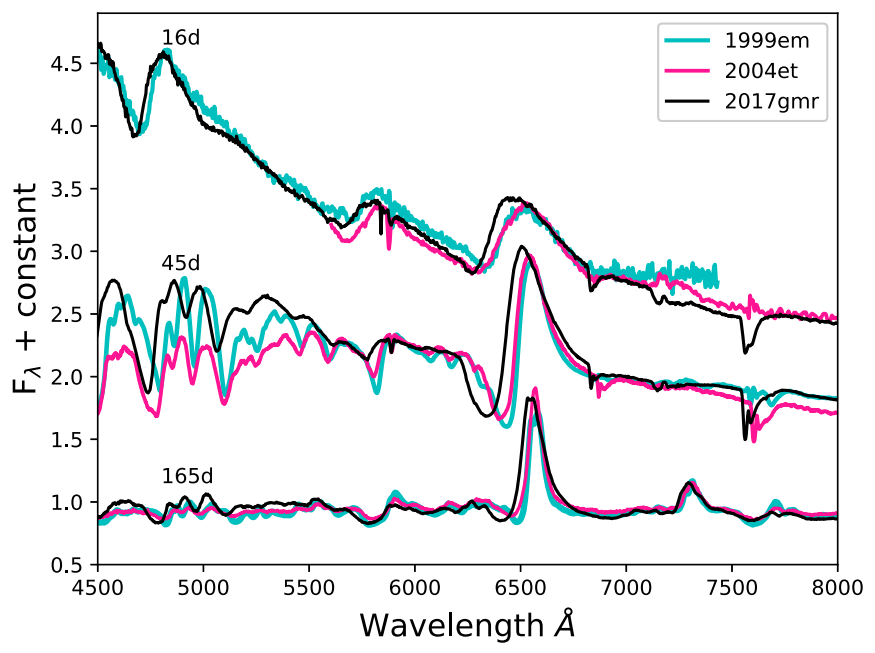

Figure 16. Comparison of SN 2017gmr with other well-studied Type II-P SNe, SN 1999em and SN 2004et, at various epochs. Data are from Leonard et al. (2002), Faran et al. (2014), and Sahu et al. (2006) and obtained from WISeREP (Yaron \& Gal-Yam 2012).

higher ${ }^{56} \mathrm{Ni}$ mass. When combined with previous conclusions of Hamuy \& Pinto (2002), Hamuy (2003), and Pejcha \& Prieto (2015), this suggests that the more energetic the explosion, the higher the luminosity, expansion velocity, and ${ }^{56} \mathrm{Ni}$ production. This may suggest that SN $2017 \mathrm{gmr}$ had an unusually energetic explosion, although low ejecta mass can also allow for high ejecta velocities.

There are other ways to create faster line velocities. If CSM interaction is occurring, it can excite $\mathrm{H} \alpha$, Fe II, and other lines at larger radii (and therefore higher velocities). This means that lines that would have otherwise already recombined in the outer, faster parts of the ejecta will be reionized and give the appearance of faster ejecta at later times. This can make it appear as if the ejecta velocities are changing slowly or stagnant, especially at later times. Faster expansion velocities can also arise from asymmetries in the explosion. Dessart \& Hillier (2011) found that asphericities in the ejecta of an axially symmetric explosion can change the location of the P Cygni minimum with inclination as much as $30 \%$ in the photospheric phase. We explore the possibility of an asymmetric explosion in Section 6.4.

\subsection{Early Narrow Features?}

Narrow lines seen within the first few days of explosion can be useful to infer composition, velocity, and density of the CSM surrounding the SN progenitor (Gal-Yam et al. 2014). One of the most well-known objects displaying this phenomenon, SN 2013fs, showed narrow $\left(\sim 100 \mathrm{~km} \mathrm{~s}^{-1}\right)$ lines of oxygen, helium, and nitrogen within the first few hours of explosion (Yaron et al. 2017; Bullivant et al. 2018). These high-excitation lines disappeared over the next 2 days, and eventually the spectra resembled those of a normal Type II SN. Similar behavior has been seen in SN 1983K (Niemela et al. 1985), SN 2006bp (Quimby et al. 2007), SN 2013cu (Gal-Yam et al. 2014), SN 1998S (Shivvers et al. 2015), PTF11iqb (Smith et al. 2015), SN 2016bkv (Hosseinzadeh et al. 2018), and SN 2014G (Terreran et al. 2016). Khazov et al. (2016) found that $14 \%-18 \%$ of their sample of Type II SNe showed signs of early narrow lines, which they conclude is a lower limit for the Type II SN population as a whole. 


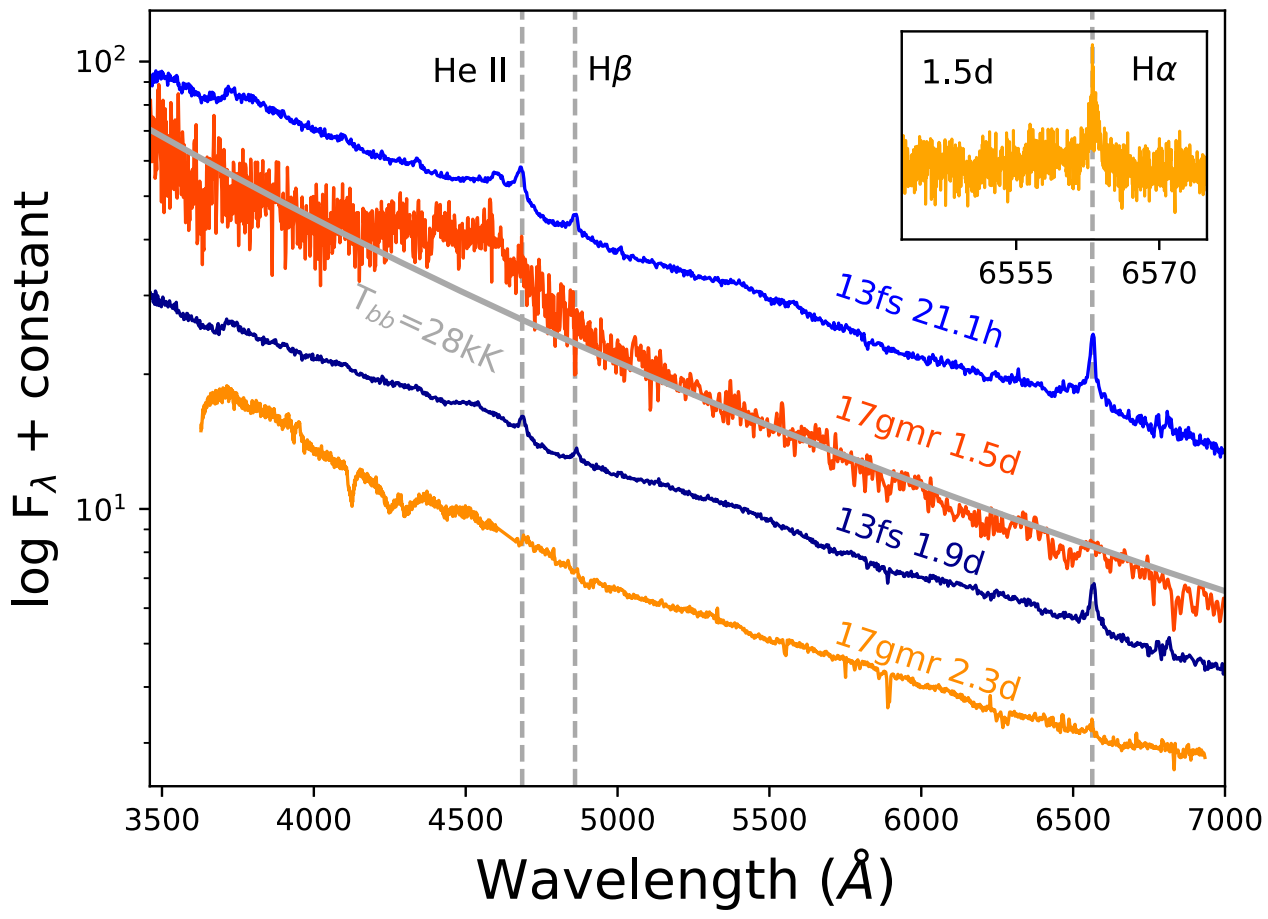

Figure 17. Comparison of the earliest spectroscopy of SN 2017gmr with that of SN 2013fs from Yaron et al. (2017). The spectrum in the inset is the 1.5-day Keck HIRES spectrum of SN $2017 \mathrm{gmr}$, which shows a weak $\mathrm{H} \alpha$ feature with a width $\sim 55 \mathrm{~km} \mathrm{~s}^{-1}$. This feature is also weakly seen in the 2.3 -day HET spectrum; the 1.5-day FLOYDS spectrum is too low resolution to identify any narrow $\mathrm{H} \alpha$. A $28 \mathrm{kK}$ blackbody is also plotted over the FLOYDS spectrum in gray.

These narrow lines were interpreted as the flash ionization of a WR-like wind for SN 2013cu (Gal-Yam et al. 2014). Later interpretation suggested that it is instead possibly the ionization of the cool dense wind from an LBV/YHG progenitor (Groh et al. 2014), which is more consistent with a Type IIb SN progenitor. Furthermore, Smith et al. (2015) found that PTF11iqb had an RSG progenitor, and the early narrow lines were likely the result of shock ionization from CSM interaction. A similar conclusion about the progenitor of SN 1998S was also reached in Shivvers et al. (2015) and Mauerhan \& Smith (2012). In other words, WR-like wind features (particularly of hydrogen-rich WNH type) can be seen in early spectra if there are enough highenergy photons to fully ionize the progenitor's cool dense wind.

SN 2017gmr was observed spectroscopically within hours after discovery, and likely within 1.5 days of shock breakout, yet the only narrow emission line seen was that of $\mathrm{H} \alpha$ (Figure 17), and only with the higher-resolution instruments. The Keck HIRES spectrum on day 1.5 (inset of Figure 17) shows a narrow $\mathrm{H} \alpha$ emission with a Gaussian FWHM velocity of $\sim 55 \mathrm{~km} \mathrm{~s}^{-1}$. This is suggestive of an RSG wind (see Smith 2014). The spectral resolution of these data is $\sim 7 \mathrm{~km} \mathrm{~s}^{-1}$, so the velocity of the ionized material is fully resolved. For reference, SN 1998S was observed with the same instrument 1.86 days after discovery and had a narrow component velocity of $\sim 40 \mathrm{~km} \mathrm{~s}^{-1}$ (Shivvers et al. 2015, albeit with lines other than $\mathrm{H} \alpha$ also present). The day 2.3 HET spectrum also seems to show a narrow but weak $\mathrm{H} \alpha$ feature with a moderately higher intermediate-width FWHM velocity of $\sim 1000 \mathrm{~km} \mathrm{~s}^{-1}$. The broadening of the line may be due to electron scattering in the CSM, and the narrow feature may be embedded within, but it has likely faded by this epoch. This feature is completely gone in the HET spectrum 3 days later; in its place is a broad, blueshifted $\mathrm{H} \alpha$ emission with an expansion velocity of $15,000 \mathrm{~km} \mathrm{~s}^{-1}$.

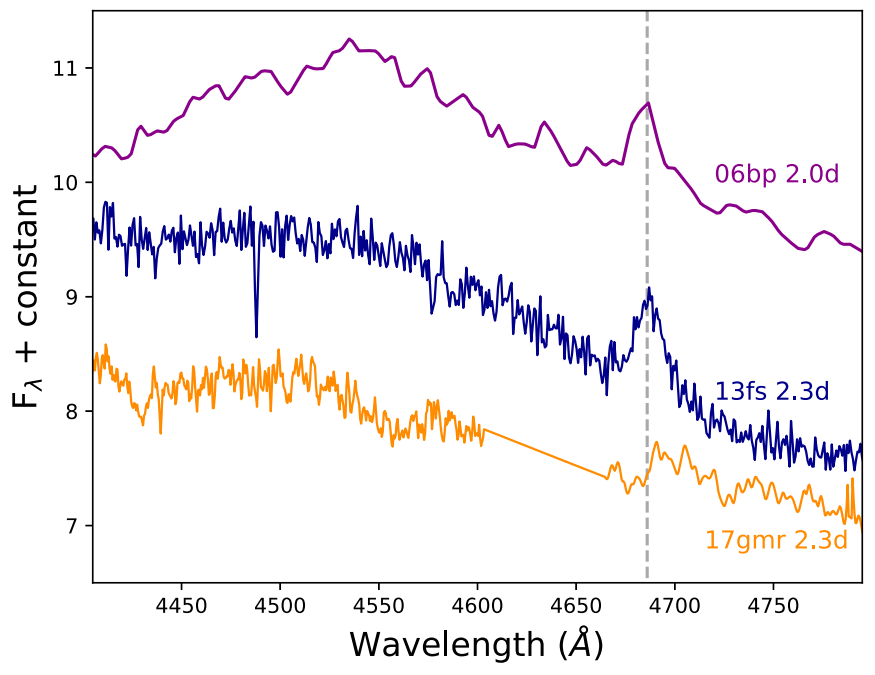

Figure 18. The 2.3-day HET spectrum (orange) of SN $2017 \mathrm{gmr}$ in the region around He II $\lambda 4686$ (dashed line) compared with the 2.3-day PESSTO spectrum of SN 2013fs (Bullivant et al. 2018) and the 2-day spectrum of SN 2006bp (Leonard 2007). None of the spectra have been corrected for reddening and have been scaled by a constant. In all three there appears to be a broad emission bump blueward of He II $\lambda 4686$.

One other noticeable feature in the very early spectra is the broad emission around $\sim 4600 \AA$ shown in detail in Figure 18. A similar broad bump was seen in SN 2006bp (Quimby et al. 2007) and SN 2013fs (Yaron et al. 2017; Bullivant et al. 2018) and was attributed to blueshifted He II $\lambda 4686$ formed from the SN ejecta beneath a CSM shell. Unfortunately, a chip gap from the HET spectrum of SN $2017 \mathrm{gmr}$ occurs just blueward of $4670 \AA$, but the overall shape is similar to that of the other SNe. SN 2006bp and SN 2013fs also showed narrow He II $\lambda 4686$ 

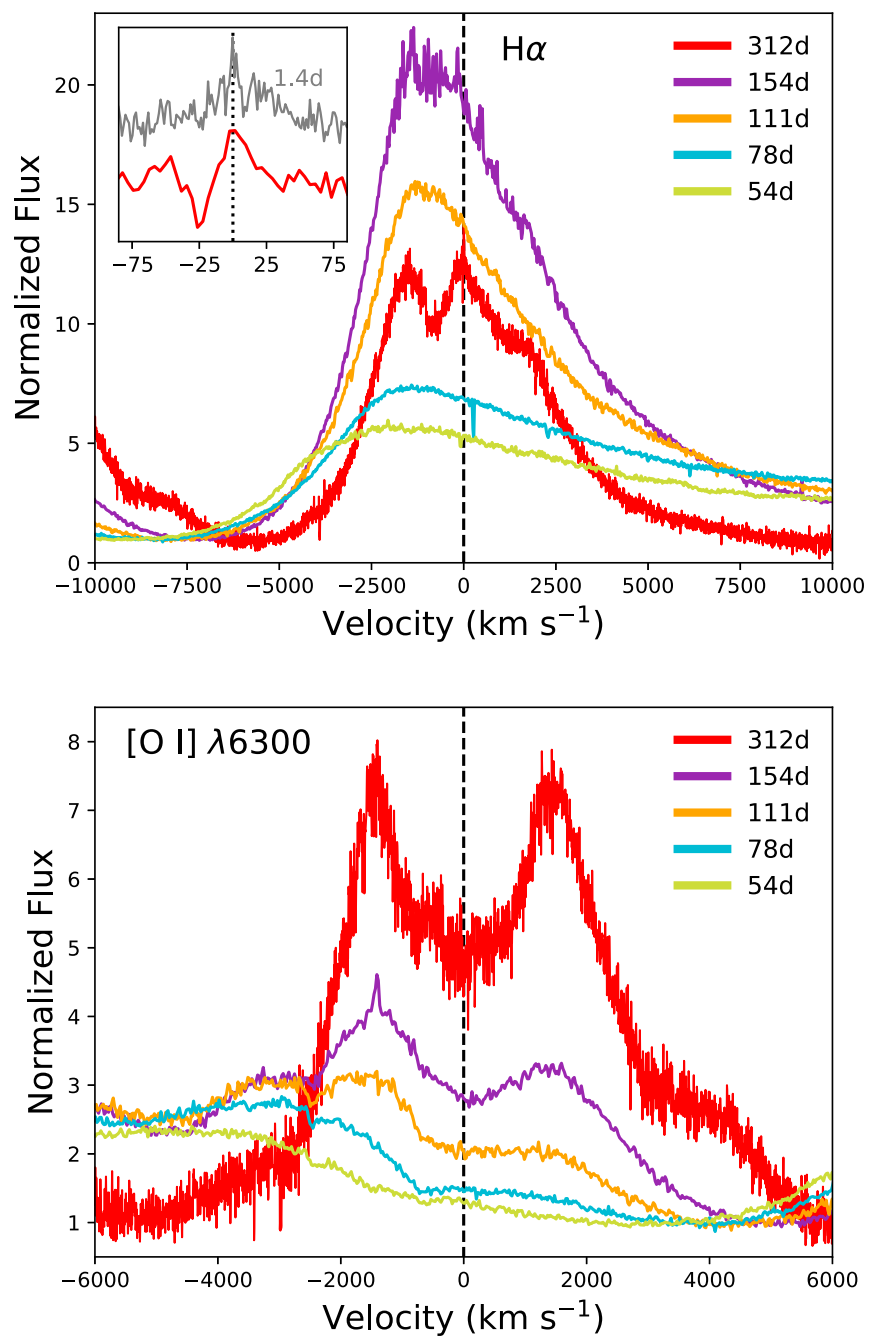

Figure 19. Evolution of the $\mathrm{H} \alpha$ (top) and [O I] $\lambda \lambda 6300,6363$ (bottom) emission lines from our moderate- and high-resolution spectra. The lines have been normalized to the minimum of the $\mathrm{H} \alpha \mathrm{P}$ Cygni line. The multipeaked shape begins to arise between 110 and 150 days but is clearly evident by our last spectrum on day 312 .

emission on the red edge of the broad $15,000 \mathrm{~km} \mathrm{~s}^{-1}$ line, which is absent in SN 2017gmr.

The lack of narrow high-ionization lines in the early spectra would seem to suggest that if nearby CSM was present, its density was too low to yield detectable emission. Alternatively, it could imply that the photons were not energetic enough to doubly ionize He in the CSM, even if SN 2017gmr likely had a very energetic explosion. If the CSM density was adequately high, this too could prevent narrow lines from forming, as it would self-absorb all of the high-energy photons. Another option would be that the narrow, high-ionization lines were present before our first spectrum at 1.5 days but were produced from asymmetric CSM, which was quickly enveloped by the spherically expanding SN ejecta (Smith et al. 2015). We will discuss this possibility further in the next section.

Another luminous Type II, SN 2016esw, was also caught within a day of explosion and showed no signs of highionization emission lines (de Jaeger et al. 2018b). The authors conclude that the progenitor of SN 2016esw was likely surrounded by low-density CSM some distance away from the surface of the star that eventually showed signs of interaction 2-3 weeks after explosion. Similarly, the Type II-P

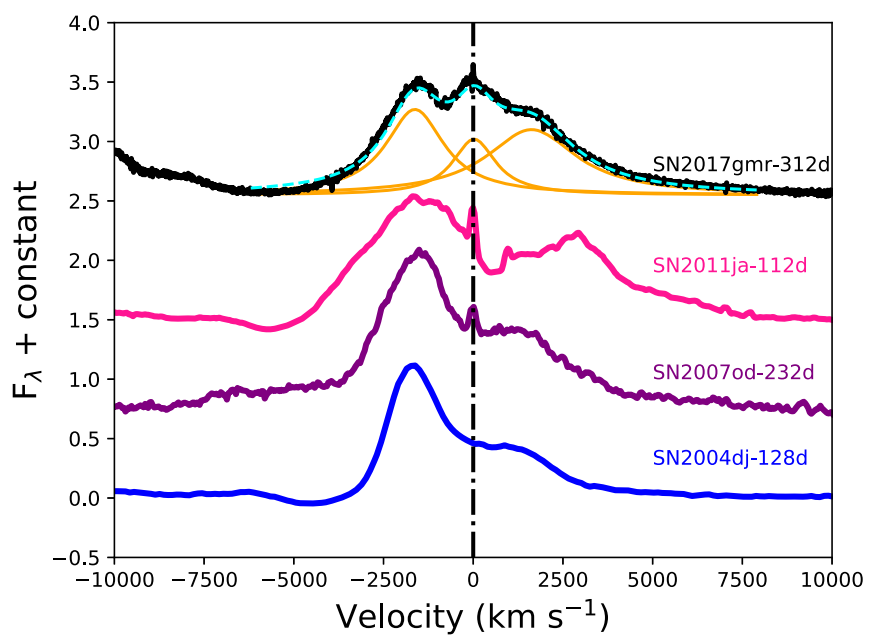

Figure 20. SN 2017gmr compared with other Type II-P SNe showing signs of CSM interaction during the radioactive tail phase. Data are from Andrews et al. (2016; SN2011ja), Andrews et al. (2010; SN2007od), and Vinkó et al. (2006; 2004dj). Lorentzian fits to the multiple components of SN 2017gmr are shown in orange, and the total fit of the separate components is overplotted as a dashed cyan line.

SN 2017eaw did not show early flash signatures (Van Dyk et al. 2019), except for possibly the $\sim 160 \mathrm{~km} \mathrm{~s}^{-1} \mathrm{H} \alpha$ line seen by Rui et al. (2019) on day 2.5. Unlike SN 2016esw, though, neither SN 2017eaw nor SN 2017gmr showed obvious signs of CSM interaction in the shape of the $\mathrm{H} \alpha$ emission line the first few weeks after explosion.

\subsection{Circumstellar Interaction or Asymmetric Explosion?}

When the SN reappeared from behind the Sun in 2018 July, we obtained one high-resolution echelle spectrum with MIKE on Magellan/Clay on day 312. The late-time analysis on SN $2017 \mathrm{gmr}$ is beyond the scope of this paper and will be discussed in depth in an upcoming paper, but due to the implications for the early-time evolution, we are including the $\mathrm{H} \alpha$ and [O I] lines here. In Figure 19 we show in red the day 312 spectrum compared to the other moderate-resolution MMT spectra. Instead of a single broad line, $\mathrm{H} \alpha$ clearly shows three intermediate-width peaks. The same is seen in the [O I] doublet, although the right peak of the $\lambda 6300$ line is stronger than the red peak of $\mathrm{H} \alpha$ due to the overlap of the blue peak from the $\lambda 6364$ line of the doublet. Signs of this asymmetry can even be seen in the day 154 spectrum.

In Figure 20 we show that the multipeaked $\mathrm{H} \alpha$ can be fit with three Lorentzians, one centered at $0 \mathrm{~km} \mathrm{~s}^{-1}(6563 \AA)$ and blue and red peaks at roughly $\pm 1700 \mathrm{~km} \mathrm{~s}^{-1}( \pm 35 \AA)$. The same velocities are seen in [O I], for both the $\lambda \lambda 6300$ and 6363 lines, but the doublet nature of the line makes it appear distinctly different. The red peak of $\lambda 6300$ would fall at $\sim 6335 \AA$, while the blue peak of $\lambda 6364$ would fall at $\sim 6330 \AA$, making the red peak of $\lambda 6300$ seem as bright as the blue peak, and swamping the emission at the center of the line.

Double-peaked emission lines in SN spectra are often interpreted as ejecta interacting with asymmetric CSM, most commonly in a disk or torus (Hoffman et al. 2008; Mauerhan et al. 2014; Smith et al. 2015; Andrews et al. 2017). In this scenario, the underlying broad component traces emission from the free expansion of the SN ejecta, while the intermediate components are formed in the post-shock region between the forward and reverse shocks created as the ejecta crashes into 
the CSM. When the fast-moving SN ejecta collides with the slow-moving CSM, depending on the density of surrounding material, the CSM can be accelerated from speeds of $10-100 \mathrm{~km} \mathrm{~s}^{-1}$ up to thousands of kilometers per second. The red and blue peaks therefore are the result of the ejecta accelerating the CSM material radially outward from the explosion. In the case of SN 2017gmr the CSM was likely accelerated from a normal RSG wind speed of $\sim 55 \mathrm{~km} \mathrm{~s}^{-1}$ to the observed intermediate feature speed of $\sim 1700 \mathrm{~km} \mathrm{~s}^{-1}$. Examples of other Type II SNe at somewhat similar phases to SN 2017gmr showing multipeaked $\mathrm{H} \alpha$ are shown in Figure 20.

The fact that we do not see narrow emission lines does not necessarily discount the possibility of SN 2017gmr being a partially CSM interaction powered event. CSM interaction can be inferred based on the intermediate-width line shapes and velocities. As explained in Smith et al. (2015), Smith (2017), and Andrews \& Smith (2018), a disk-like geometry in the CSM may allow the CSM interaction to be hidden below the photosphere after the disk is enveloped by the fast SN ejecta. If the region of CSM interaction is happening below the ejecta photosphere and the CSM is sufficiently dense, it can be hidden for long periods of time because the sustained CSM interaction luminosity itself keeps the surrounding SN ejecta ionized and optically thick. This could help explain the extended high luminosity of SN $2017 \mathrm{gmr}$. All that is required is that the disk or torus of material has a limited radial extent (i.e., $\leqslant 100 \mathrm{au}$ ) so that it can be overrun early by the SN photosphere. Only when the photosphere recedes internal to the CSM location (which has been pushed outward to $1700 \mathrm{~km} \mathrm{~s}^{-1}$ owing to the Doppler acceleration) will the intermediate-width lines be revealed.

If we assume that high-ionization lines were observable prior to our 1.5-day spectrum, we can use the expansion velocity of $\mathrm{H} \alpha\left(15,000 \mathrm{~km} \mathrm{~s}^{-1}\right)$ to infer that the outer edge of the CSM must be closer than $1.8 \times 10^{14} \mathrm{~cm}$ (or $\sim 2500 R_{\odot}$ ). This is roughly the same radius inferred for SN 2013cu (Gal-Yam et al. 2014) and PTF11iqb (Smith et al. 2015).

The other possibility is that the multiple peaks seen in the emission lines could come from asymmetries in the ejecta (Maeda et al. 2008; Taubenberger et al. 2009). This was the scenario presented for SN 2003jd (Mazzali et al. 2005), SN 2004dj (Chugai et al. 2005, shown in Figure 20), SN 2010jp (Smith et al. 2012a), and SN 2016X (Huang et al. 2018; Bose et al. 2019). In a forthcoming paper Nagao et al. (2019) find that there is strong polarization in SN 2017gmr indicative of an aspherical explosion. Nonuniformity of ${ }^{56} \mathrm{Ni}$ could cause uneven ionization and excitation in the ejecta and produce multipeaked emission lines. SN 2004dj showed strong $\mathrm{H} \alpha$ asymmetry immediately after the plateau phase ended, during the epoch of increased polarization (Leonard et al. 2006). As we show in Figure 19, distinct multiple peaks are not present until sometime between 154 and 312 days, or a significant time period after the end of the plateau. Also of note is that there is a component at rest velocity at late times in SN 2017gmr that would have to come from some spherically distributed radioactive material.

In general, it is difficult to disentangle the two mechanisms. The low polarization at early times is explained by Nagao et al. (2019) as the hydrogen envelope hiding a highly asymmetric helium core that is only observable when the optical depth decreases. We suggest that it could also be explained partially (or in full) by the spherical symmetry of the hydrogen envelope erasing the polarization signatures of deeply embedded asymmetric CSM interaction. The deviation from ${ }^{56}$ Co decay in the late-time light curve can be due to incomplete $\gamma$ photon trapping caused by a nonspherical ejecta, or it could be due to a decrease in the shock interaction. Whatever the mechanism, the emission-line shapes emerging during the nebular phase indicate a deviation from spherical symmetry, whether it be from asymmetric stellar ejecta or shock interaction with a disk or torus of CSM.

\subsection{Dust Formation?}

As we briefly mention above, the optical and bolometric light curves decline faster than 0.98 mag 100 day $^{-1}$ attributed to ${ }^{56} \mathrm{Co}$ decay. The fast decline could indicate the halting of shock interaction as a primary energy source, or that there is incomplete trapping of gamma-rays as we discuss in Section 6.1. It could also be due in all, or part, to dust formation in the ejecta.

Along with a decrease in optical luminosity from the growth of dust grains, we can also expect to see a blueshifted asymmetry in the optical emission lines since dust in the ejecta would attenuate the receding red side of the SN more than the blue. First detected in SN 1987A (Lucy et al. 1989), evidence for dust formation has been seen in many CCSNe, including SN 2003gd (Sugerman et al. 2006), SN 2004et (Kotak et al. 2009), SN 2005ip (Smith et al. 2009; Fox et al. 2010; Stritzinger et al. 2012; Bevan et al. 2019), SN 2006jd (Stritzinger et al. 2012), SN 2007od (Andrews et al. 2010; Inserra et al. 2011), SN 2010j1 (Smith et al. 2012b; Gall et al. 2014), and one of the clearest cases, SN 2006jc (Mattila et al. 2008; Smith et al. 2008). In conjunction with the emission-line asymmetry and a decrease in the optical light curve, a corresponding increase in the IR luminosity is often observed as new dust grains form in the ejecta.

It is unlikely that dust has formed in SN 2017gmr by $\sim 150$ days for a few reasons. First, a blackbody fit to the optical and NIR spectroscopy and photometry around day 150 indicates $T_{\mathrm{bb}}=6800 \mathrm{~K}$, a temperature much too high for grain condensation. Second, the bolometric light curve also shows a deviation from expected ${ }^{56} \mathrm{Co}$ decay. If dust formation was occurring, the light curves in individual bands will change, but the total bolometric light curve would be unchanged. Finally, as we mention above, the NIR spectroscopy during the early nebular phase fail to reveal the first overtone of CO (Figure 13). Normally the detection of $\mathrm{CO}$ heralds the formation of dust (Gerardy et al. 2000; Sarangi \& Cherchneff 2013). Therefore, the blue-peaked hydrogen emission profiles and the fast decline in $L_{\text {bol }}$ are likely due to other physical characteristics of the SN such as asymmetries and CSM interaction, not dust formation. This does not discount the possibility that in later epochs we may begin to see signatures of dust condensation in the ejecta.

\section{Conclusions}

SN 2017gmr is one of the more luminous Type II-P SNe discovered to date, with one of the largest measured ${ }^{56} \mathrm{Ni}$ masses for a Type II-P event. Not only does it peak at $M_{V}=-18.3 \mathrm{mag}$, but by 150 days after explosion it has declined less than $3 \mathrm{mag}$ in the $V$ band. If the late-time luminosity is powered solely by radioactive decay, then the mass of ${ }^{56} \mathrm{Ni}$ is $0.130 \pm 0.026 M_{\odot}$, quite massive for a Type II$\mathrm{P}$ SN. The line velocities are abnormally fast for a Type II-P event, which could be due to an extremely energetic explosion, 
asymmetries in the ejecta, or CSM interaction reionizing the faster, outer parts of the ejecta. The inferred progenitor radius is $\sim 500 R_{\odot}$, on the lower end for an RSG, but within normally expected values.

CSM interaction is an efficient way to convert SN ejecta kinetic energy into radiative luminosity. The high luminosity of SN 2017gmr at late times and the bump in the early-time $U$ and $B$ light curves could both be the result of an added energy contribution from CSM interaction. The fact that no narrow lines are seen at early times could be due to the spherical ejecta quickly overtaking the asymmetric CSM, and the lack of narrow lines at late times only indicates that the $\mathrm{SN}$ shock has moved completely through the close-in CSM. In other words, all the slow-moving CSM has been swept up by the shock. Low polarization during the plateau phase (Nagao et al. 2019) could also be explained by mostly spherical ejecta enveloping a dense, close-in asymmetric CSM. Since these CSM interaction photons are thermalized deep inside the opaque SN ejecta envelope, their polarization signature from asymmetric CSM would be erased. Asymmetric explosions producing jets or blobs of ${ }^{56} \mathrm{Ni}$ could also create the asymmetric emission lines and the high line velocities.

SN $2017 \mathrm{gmr}$ was caught very young, and the collection of high-cadence multiwavelength data began immediately. This has allowed us the ability to explore not only the early behavior of Type II SNe but the years of mass loss prior to explosion. More instances of early data are needed to understand both this mass loss and the diversity among $\mathrm{SNe}$ in these early-time properties. Either SN 2017gmr is an unusually energetic Type II-P SN explosion, or it has the assistance of CSM interaction and asymmetries to make it appear so. Continued observations of SN 2017gmr are ongoing and are necessary to help disentangle the various energy inputs and the overall geometry of this unique event.

We acknowledge contributions from S. Taubenberger, T. Nagao, and N. Suntzeff. J.E.A. and N.S. received support from NSF grant AST-1515559. Research by D.J.S. and S.W. is supported by NSF grants AST-1821987 and 1821967. Research by S.V. is supported by NSF grants AST-1813176. G.H., D.A.H., and C.M. were supported by NSF grant AST1313484. K.M. acknowledges the support from the Department of Science and Technology (DST), Govt. of India and Indo-US Science and Technology Forum (IUSSTF) for the WISTEMM fellowship and the Department of Physics, UC Davis, where a part of this work was carried out. G.H. thanks the LSSTC Data Science Fellowship Program, which is funded by LSSTC, NSF Cybertraining grant No. 1829740, the Brinson Foundation, and the Moore Foundation; his participation in the program has benefited this work. J.V. and his group at Konkoly are supported by the project "Transient Astrophysical Objects" GINOP-2-3-2-15-2016-00033 of the National Research, Development and Innovation Office (NKFIH), Hungary, funded by the European Union. L.W. is sponsored (in part) by the Chinese Academy of Sciences (CAS), through a grant to the CAS South America Center for Astronomy (CASSACA) in Santiago, Chile. M.B. acknowledges support from the Swedish Research Council (Vetenskapsrådet), the Swedish National Space Board, and the research environment grant "Gravitational Radiation and Electromagnetic Astrophysical Transients (GREAT)." J.S. acknowledges support from the Packard Foundation. G.P. and O.R. acknowledge support by projects IC120009 "Millennium
Institute of Astrophysics (MAS)" of the Iniciativa Científica Milenio del Ministerio Economía, Fomento y Turismo de Chile and CONICYT PAI/INDUSTRIA 79090016. B.K. also acknowledges the Science and Engineering Research Board (SERB) under the Department of Science \& Technology, Govt. of India, for financial assistance in the form of National PostDoctoral Fellowship (Ref. no. PDF/2016/001563). Research by J.C.W. is supported by NSF AST-1813825. Support for F.O.E. is provided by the Ministry of Economy, Development, and Tourism's Millennium Science Inititative through grant IC120009, awarded to the Millennium Institute of Astrophysics, MAS. K.M. acknowledges support from H2020 through an ERC Starting Grant (758638). F.O.E. acknowledges support from the FONDECYT grant 11170953. M.G. is supported by the Polish NCN MAESTRO grant 2014/14/A/ST9/00121. S.B. is partially supported by PRIN INAF 2017 "Towards the SKA and CTA era: discovery, localisation and physics of transient sources (PI M. Giroletti)." M.D.S. is funded by a project grant (8021-00170B) from the Independent Research Fund Denmark and also in part by a generous grant (13261) from VILLUM FONDEN. N.E.R. acknowledges support from the Spanish MICINN grant ESP2017-82674-R and FEDER funds. E.Y.H., C.A., S.D. and M.S. acknowledge the support provided by the National Science Foundation under grant No. AST-1613472. C.G. was supported by a grant from VILLUM FONDEN (project No. 16599). C.G. was supported by a grant from VILLUM FONDEN (project No. 16599). X.W. is supported by the National Natural Science Foundation of China (NSFC grants 11325313, 11633002, and 11761141001) and the National Program on Key Research and Development Project (grant No. 2016YFA0400803). This work was also partially supported by the Open Project Program of the Key Laboratory of Optical Astronomy, National Astronomical Observatories, Chinese Academy of Sciences. NUTS is supported in part by IDA (Instrumentation center for Danish Astrophysics).

Observations reported here were obtained at the MMT Observatory, a joint facility of the University of Arizona and the Smithsonian Institution. This work makes use of data obtained by the Las Cumbres Observatory network. Based on observations obtained at the Gemini Observatory under program GN-2017B-Q-52 (PI: Sand), which is operated by the Association of Universities for Research in Astronomy, Inc., under a cooperative agreement with the NSF on behalf of the Gemini partnership: the National Science Foundation (United States), National Research Council (Canada), CONICYT (Chile), Ministerio de Ciencia, Tecnología e Innovación Productiva (Argentina), Ministério da Ciência, Tecnologia e Inovação (Brazil), and Korea Astronomy and Space Science Institute (Republic of Korea). Based in part on observations collected at Copernico and Schmidt telescopes (Asiago, Italy) of the INAF-Osservatorio Astronomico di Padova. This paper is partially based on observations collected with the $1.22 \mathrm{~m}$ Galileo telescope of the Asiago Astrophysical Observatory, operated by the Department of Physics and Astronomy "G. Galilei" of the University of Padova. Based on observations made with the Nordic Optical Telescope, operated by the Nordic Optical Telescope Scientific Association at the Observatorio del Roque de los Muchachos, La Palma, Spain, of the Instituto de Astrofisica de Canarias. Based on observations obtained at the Southern Astrophysical Research (SOAR) telescope, which is a joint project of the Ministério da 
Ciência, Tecnologia, Inovações e Comunicações (MCTIC) do Brasil, the U.S. National Optical Astronomy Observatory (NOAO), the University of North Carolina at Chapel Hill (UNC), and Michigan State University (MSU). Partially based on observations collected at the European Southern Observatory under ESO program 099.D-0543(A). We thank the staff of IAO, Hanle and CREST, Hosakote, that made the observations from HCT possible. The facilities at IAO and CREST are operated by the Indian Institute of Astrophysics, Bangalore.

The authors wish to recognize and acknowledge the very significant cultural role and reverence that the summit of Maunakea has always had within the indigenous Hawaiian community. We are most fortunate to have the opportunity to conduct observations from this mountain. Some of the data presented herein were obtained at the W. M. Keck Observatory, which is operated as a scientific partnership among the California Institute of Technology, the University of California, and the National Aeronautics and Space Administration. The Observatory was made possible by the generous financial support of the W. M. Keck Foundation, which is operated by the University of Hawaii under contract NNH14CK55B with the National Aeronautics and Space Administration. This publication makes use of data products from the Two Micron All Sky Survey, which is a joint project of the University of Massachusetts and the Infrared Processing and Analysis Center/California Institute of Technology, funded by the National Aeronautics and Space Administration and the National Science Foundation. We made use of Swift/UVOT data reduced by P. J. Brown and released in the Swift Optical/ Ultraviolet Supernova Archive (SOUSA). SOUSA is supported by NASAs Astrophysics Data Analysis Program through grant NNX13AF35G.

Facilities: ARIES:ST, ARIES:DFOT, CTIO:PROMPT, Las Cumbres Observatory (FLOYDS, Sinistro), Gemini:Gillett (GNIRS), IRTF (SpeX), Magellan:Baade (FIRE), Magellan: Clay (MIKE), ING:Newton (IDS), NTT (EFOSC2, SOFI), MMT (BCS), Keck:I (HIRES), Beijing:2.16 m (BFOSC), NOT (ALFOSC), SOAR (Goodman), Asiago:Copernico (AFOSC), Asiago:Galileo (B\&C), VLT:Antu (FORS2), HCT (HFOSC), HET(LRS2), SO:Super-LOTIS, Bok (B\&C), Liverpool:2 m (IO:O), REM.

Software: astropy (Astropy Collaboration et al. 2013; The Astropy Collaboration et al. 2018), LCOGTSNPIPE (Valenti et al. 2016), superbol (Nicholl 2018).

\section{Appendix A \\ Photometry}

\section{A.1. UV and Optical}

Photometric data for SN 2017gmr was obtained from a variety of telescopes (see Section 2), resulting in an extremely high cadence optical light curve (Figure 4), as well as an earlytime Swift UV+optical light curve (Figure 6). We briefly describe the instrumentation and data reduction techniques here, although if a telescope+instrument combination is not specifically mentioned, the data were reduced in a "standard" way, including image detrending (bias subtraction and flatfielding), cosmic-ray removal, point-spread function (PSF) or aperture photometry, along with flux calibration performed against standard catalogs (e.g., Landolt standard stars or the Sloan Digital Sky Survey [SDSS]). The full ground-based
Table 1

SN 2017gmr Optical Photometry

\begin{tabular}{lcccc}
\hline \hline MJD & Phase & Magnitude & Error & Telescope \\
\hline & & $U$ & & \\
\hline $58,000.276$ & +1.19 & 14.44 & 0.05 & LCO-1 m \\
$58,000.280$ & +1.19 & 14.37 & 0.04 & LCO-1 m \\
$58,000.358$ & +1.27 & 14.11 & 0.02 & LCO-1 m \\
$58,000.362$ & +1.27 & 14.11 & 0.02 & LCO-1 m \\
$58,000.631$ & +1.54 & 13.90 & 0.03 & LCO-1 m \\
$58,000.635$ & +1.54 & 13.90 & 0.03 & LCO-1 m \\
$58,000.984$ & +1.89 & 13.87 & 0.05 & LCO-1 \\
$58,000.987$ & +1.89 & 13.94 & 0.03 & LCO-1 m \\
$58,001.115$ & +2.03 & 13.89 & 0.03 & LCO-1 m \\
$58,001.119$ & +2.03 & 13.91 & 0.03 & LCO-1 m \\
\hline
\end{tabular}

Note. Phases are reported with respect to an assumed explosion epoch of MJD 57,999.09.

(This table is available in its entirety in machine-readable form.)

optical data set is presented in Table 1, while the Swift data are presented in Table 2.

First, continued monitoring of SN 2017gmr was done by the DLT40 survey's two discovery telescopes, the PROMPT5 $0.4 \mathrm{~m}$ telescope at Cerro Tololo Inter-American Observatory and the PROMPT-MO $0.4 \mathrm{~m}$ telescope at Meckering Observatory in Australia, operated by the Skynet telescope network (Reichart et al. 2005). The PROMPT5 telescope has no filter ("Open"), while the PROMPT-MO telescope has a broadband "Clear" filter, both of which we calibrate to the SDSS $r$ band (see Tartaglia et al. 2018, for further reduction details).

Las Cumbres Observatory UBVgri-band data were obtained with the Sinistro cameras on the $1 \mathrm{~m}$ telescopes, through the Global Supernova Project. Using LCOGTSNPIPE (Valenti et al. 2016), a PyRAF-based photometric reduction pipeline, PSF fitting was performed. $U B V$-band data were calibrated to Vega magnitudes (Stetson 2000) using standard fields observed on the same night by the same telescope. Finally, gri-band data were calibrated to $\mathrm{AB}$ magnitudes using the SDSS (SDSS Collaboration et al. 2017). Because the Las Cumbres data are the most comprehensive, and there are differences across the instrument/filter pairs, all other data sets were shifted by small amounts to match the Las Cumbres magnitudes in Figure 4. These values ranged between 0.05 and $0.15 \mathrm{mag}$. The nonshifted values are all included in Table 1.

Optical photometry in the BVRI bands was obtained at the $60 / 90 \mathrm{~cm}$ Schmidt telescopes at Konkoly Observatory; see Vinkó et al. (2012) for a description of the instrumentation and data reduction techniques. Further, BVRI photometry was obtained with the Super-LOTIS (Livermore Optical Transient Imaging System; Williams et al. 2008) $0.6 \mathrm{~m}$ telescope at Kitt Peak National Observatory; these data were reduced in a manner similar to that described in Kilpatrick et al. (2016), and PSF photometry using standard IRAF procedures was then done on the resultant images.

Data in the BVugriz bands were taken with the IO:O imager on the Liverpool telescope and were reduced using the standard IO:O pipeline; aperture photometry was performed using custom PYTHON scripts and PYRAF. The data were shifted $+0.17 \mathrm{mag}$ in $B$ to match the Las Cumbres data. Data from the $1.30 \mathrm{~m}$ DFOT and 2.01 HCT telescopes were reduced as described in Dastidar et al. (2019) performing PSF fitting photometry using DAOPHOT II (Stetson 1987). Instrumental 
Table 2

SN 2017gmr Swift Photometry

\begin{tabular}{|c|c|c|c|}
\hline MJD & Phase & Magnitude & $\overline{\text { Error }}$ \\
\hline \multicolumn{4}{|c|}{$U V W 2$} \\
\hline $58,001.626$ & +2.5 & 13.80 & 0.07 \\
\hline $58,002.767$ & +3.7 & 14.05 & 0.07 \\
\hline $58,003.565$ & +4.5 & 14.26 & 0.20 \\
\hline $58,008.442$ & +9.3 & 15.26 & 0.08 \\
\hline \multicolumn{4}{|c|}{ UVM2 } \\
\hline $58,001.606$ & +2.5 & 13.97 & 0.15 \\
\hline $58,002.771$ & +3.7 & 14.20 & 0.06 \\
\hline $58,003.569$ & +4.5 & 14.40 & 0.06 \\
\hline $58,008.313$ & +9.2 & 15.03 & 0.07 \\
\hline \multicolumn{4}{|c|}{$U V W 1$} \\
\hline $58,001.622$ & +2.5 & 13.60 & 0.07 \\
\hline $58,002.764$ & +3.7 & 13.71 & 0.08 \\
\hline $58,003.562$ & +4.5 & 13.78 & 0.07 \\
\hline $58,008.441$ & +9.3 & 14.27 & 0.20 \\
\hline \multicolumn{4}{|c|}{$u$} \\
\hline $58,001.623$ & +2.5 & 13.58 & 0.05 \\
\hline $58,002.766$ & +3.7 & 13.60 & 0.05 \\
\hline $58,003.563$ & +4.5 & 13.62 & 0.05 \\
\hline $58,008.442$ & +9.3 & 13.70 & 0.05 \\
\hline \multicolumn{4}{|c|}{$b$} \\
\hline $58,001.624$ & +2.5 & 14.81 & 0.30 \\
\hline $58,002.766$ & +3.7 & 14.70 & 0.14 \\
\hline $58,003.563$ & +4.5 & 14.65 & 0.12 \\
\hline $58,008.442$ & +9.3 & 14.62 & 0.10 \\
\hline \multicolumn{4}{|c|}{$v$} \\
\hline $58,001.596$ & +2.5 & 14.52 & 0.08 \\
\hline $58,002.769$ & +3.7 & 14.43 & 0.07 \\
\hline $58,003.567$ & +4.5 & 14.51 & 0.07 \\
\hline $58,008.312$ & +9.2 & 14.25 & 0.17 \\
\hline
\end{tabular}

Note. Phases are reported with respect to an assumed explosion epoch of MJD $57,999.09$.

magnitudes were converted to standard magnitudes using a set of local standard stars and observations of either Landolt standard or SDSS fields.

The Swift UVOT analysis uses the pipeline of the Swift Optical/Ultraviolet Supernova Archive ${ }^{52}$ (SOUSA; Brown et al.

2014). The method is based on that of Brown et al. (2009), including subtraction of the host galaxy count rates, and uses the revised UV zero-points and time-dependent sensitivity from Breeveld et al. (2011). For SN 2017gmr we do not have template images to subtract the underlying galaxy flux. In this case, however, the largest contributor to the background is scattered/reflected light from the nearby bright star evident in Figure 1. The reported UVOT magnitudes use a background position that to the eye approximated the brightness of the galaxy and halo at the SN position. The errors have been conservatively increased to match the range of magnitudes measured with a variety of halo-free and bright halo regions. The full Swift data set is presented in Table 2 and is plotted in Figure 6.

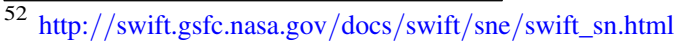

Table 3

NIR Photometry

\begin{tabular}{lccc}
\hline \hline MJD & $J$ & $H$ & $K$ \\
\hline \multicolumn{3}{c}{ NOT } \\
\hline $58,005.5$ & $13.84 \pm 0.05$ & $13.77 \pm 0.07$ & $13.94 \pm 0.12$ \\
$58,025.3$ & $13.13 \pm 0.05$ & $12.91 \pm 0.07$ & $12.70 \pm 0.12$ \\
$58,042.5$ & $13.08 \pm 0.05$ & $12.84 \pm 0.07$ & $12.86 \pm 0.12$ \\
$58,121.1$ & $14.43 \pm 0.05$ & $14.18 \pm 0.07$ & $14.19 \pm 0.12$ \\
$58,140.5$ & $14.72 \pm 0.05$ & $14.63 \pm 0.07$ & $14.67 \pm 0.12$ \\
$58,165.8$ & $15.27 \pm 0.05$ & $15.19 \pm 0.07$ & $15.23 \pm 0.12$ \\
\hline & \multicolumn{3}{c}{ REM } \\
\hline $58,012.26$ & $13.23 \pm 0.03$ & $13.09 \pm 0.03$ & $12.88 \pm 0.06$ \\
$58,017.32$ & $13.23 \pm 0.03$ & $13.05 \pm 0.03$ & $12.87 \pm 0.06$ \\
$58,021.31$ & $13.26 \pm 0.03$ & $12.96 \pm 0.03$ & $12.84 \pm 0.03$ \\
$58,022.31$ & $14.21 \pm 0.03$ & $12.97 \pm 0.03$ & $12.77 \pm 0.22$ \\
$58,027.25$ & $13.03 \pm 0.04$ & $12.81 \pm 0.04$ & $\cdots$ \\
$58,073.12$ & $13.09 \pm 0.04$ & $12.79 \pm 0.05$ & $\cdots$ \\
$58,083.06$ & $13.23 \pm 0.03$ & $12.98 \pm 0.04$ & $\cdots$ \\
$58,093.07$ & $13.59 \pm 0.03$ & $13.35 \pm 0.04$ & $\cdots$ \\
$58,109.21$ & $14.28 \pm 0.04$ & $14.05 \pm 0.05$ & $\cdots$ \\
\hline
\end{tabular}

\section{A.2. Near-infrared}

Raw NIR data from NOTCam were reduced using the NOTCam Quicklook reduction package, and PSF photometry was then performed using standard IRAF procedures. The REM telescope is equipped with an optical and an IR camera, which observes simultaneously the same field, thanks to a dichroic placed before the telescope focal plane. IR images were corrected for dark current and flat-fielded, and subsequently median-stacked to obtain a background frame for each filter. The background-subtracted images were geometrically aligned and then stacked to obtain a final image for each filter, and the background in the locations of SN 2017gmr was modeled with a low-order polynomial surface and subtracted. The flux of the SN and the local sequence were measured through PSF fitting. For both instruments, photometric calibration was done using Two Micron All Sky Survey stars in the field. The resulting data set can be found in Table 3.

\section{Appendix B Spectroscopy}

\section{B.1. Optical Spectroscopy}

A high-cadence spectral sequence of SN $2017 \mathrm{gmr}$ was taken with low-, medium-, and high-resolution instrumentation throughout the rise, plateau, and fall from plateau of the SN. A $\log$ of these observations can be found in Table 4. These spectra were reduced using standard techniques, including bias subtraction, flat-fielding, cosmic-ray rejection, local sky subtraction, and extraction of one-dimensional spectra. Most observations had the slit aligned along the parallactic angle to minimize differential light losses. Flux calibration was done with standard-star observations, and most spectra were rescaled to match the photometric light curve at a given epoch. We discuss some details of the spectroscopic reductions below, but if a particular telescope+instrument combination is not mentioned, it was reduced in a standard way as described above.

Las Cumbres optical spectra were taken with the FLOYDS spectrographs mounted on the $2 \mathrm{~m}$ Faulkes Telescope North 
Table 4

Optical Spectroscopy of SN 2017gmr

\begin{tabular}{|c|c|c|c|c|c|}
\hline $\begin{array}{l}\text { UT Date } \\
(\mathrm{y} \mathrm{m} \mathrm{d})\end{array}$ & MJD & $\begin{array}{l}\text { Phase } \\
\text { (days) }\end{array}$ & $\begin{array}{c}\text { Telescope }+ \\
\text { Instrument }\end{array}$ & $\begin{array}{c}R \\
\lambda / \Delta_{\lambda}\end{array}$ & $\begin{array}{c}\text { Exposure Time } \\
\text { (s) }\end{array}$ \\
\hline 2017 Sep 4 & $58,000.57$ & 1.5 & FTS+FLOYDS & 500 & 2700 \\
\hline 2017 Sep 4 & $58,000.59$ & 1.5 & Keck+HIRES & 50000 & $3 \times 900$ \\
\hline 2017 Sep 5 & $58,001.34$ & 2.2 & $\mathrm{SOAR}+$ Goodman & 500 & 900 \\
\hline 2017 Sep 5 & $58,001.43$ & 2.3 & HET+LRS2B & 1100 & 1000 \\
\hline 2017 Sep 5 & $58,001.62$ & 2.5 & FTS+FLOYDS & 500 & 2700 \\
\hline 2017 Sep 6 & $58,002.19$ & 3.1 & INT+IDS & 300 & $2 \times 900$ \\
\hline 2017 Sep 8 & $58,004.19$ & 5.1 & INT+IDS & 300 & 1200 \\
\hline 2017 Sep 8 & $58,004.35$ & 5.3 & Mag+IMACS & 4000 & 300 \\
\hline 2017 Sep 8 & $58,004.44$ & 5.4 & $\mathrm{HET}+\mathrm{LRS} 2 \mathrm{~B}$ & 1100 & 1000 \\
\hline 2017 Sep 8 & $58,004.55$ & 5.5 & $\mathrm{HCT}+\mathrm{HFOSC}$ & 350 & $2 \times 1200$ \\
\hline 2017 Sep 8 & $58,004.56$ & 5.5 & FTS+FLOYDS & 500 & 2700 \\
\hline 2017 Sep 10 & $58,006.19$ & 7.1 & $\mathrm{NOT}+\mathrm{ALFOSC}$ & 300 & 900 \\
\hline 2017 Sep 10 & $58,006.76$ & 7.7 & FTS+FLOYDS & 500 & 2700 \\
\hline 2017 Sep 11 & $58,007.31$ & 8.2 & $\mathrm{NTT}+\mathrm{EFOSC} 2$ & 200 & 600 \\
\hline 2017 Sep 11 & $58,007.41$ & 8.3 & HET+LRS2B & 1100 & 1000 \\
\hline 2017 Sep 12 & $58,008.50$ & 9.4 & FTN+FLOYDS & 500 & 2700 \\
\hline 2017 Sep 12 & $58,008.50$ & 9.4 & Bok + BC & 700 & $3 \times 120$ \\
\hline 2017 Sep 15 & $58,011.47$ & 12.4 & FTN+FLOYDS & 500 & 2700 \\
\hline 2017 Sep 16 & $58,012.13$ & 13.0 & $\mathrm{NOT}+\mathrm{ALFOSC}$ & 300 & $2 \times 300$ \\
\hline 2017 Sep 16 & $58,012.69$ & 13.6 & FTS+FLOYDS & 500 & 2700 \\
\hline 2017 Sep 18 & $58,014.53$ & 15.4 & FTN+FLOYDS & 500 & 2700 \\
\hline 2017 Sep 19 & $58,015.78$ & 16.7 & $\mathrm{BAO}+\mathrm{BFOSC}$ & 500 & 2400 \\
\hline 2017 Sep 22 & $58,018.06$ & 19.0 & $\mathrm{NOT}+\mathrm{ALFOSC}$ & 300 & $2 \times 300$ \\
\hline 2017 Sep 22 & $58,018.69$ & 19.6 & $\mathrm{BAO}+\mathrm{BFOSC}$ & 500 & 2400 \\
\hline 2017 Sep 25 & $58,021.42$ & 22.3 & FTN +FLOYDS & 500 & 2700 \\
\hline 2017 Sep 26 & $58,022.78$ & 23.7 & $\mathrm{BAO}+\mathrm{BFOSC}$ & 500 & 2400 \\
\hline 2017 Sep 27 & $58,024.00$ & 24.9 & $\mathrm{NOT}+\mathrm{ALFOSC}$ & 300 & $2 \times 300$ \\
\hline 2017 Sep 29 & $58,025.40$ & 26.4 & $\mathrm{Bok}+\mathrm{BC}$ & 700 & $3 \times 600$ \\
\hline 2017 Oct-3 & $58,029.53$ & 30.4 & FTN+FLOYDS & 500 & 2700 \\
\hline 2017 Oct-5 & $58,031.06$ & 32.0 & $\mathrm{NOT}+\mathrm{ALFOSC}$ & 300 & 600 \\
\hline 2017 Oct-11 & $58,037.13$ & 38.0 & $\mathrm{NOT}+\mathrm{ALFOSC}$ & 300 & 600 \\
\hline 2017 Oct-11 & $58,037.40$ & 38.3 & Bok + BC & 700 & $3 \times 240$ \\
\hline 2017 Oct-17 & $58,043.01$ & 43.9 & Asiago $182+$ AFOSC & 300 & $2 \times 1200$ \\
\hline 2017 Oct-17 & $58,043.25$ & 44.2 & VLT+FORS2 & 500 & $274+343$ \\
\hline 2017 Oct-18 & $58,044.74$ & 45.7 & $\mathrm{HCT}+\mathrm{HFOSC}$ & 350 & $2 \times 1200$ \\
\hline 2017 Oct-18 & $58,044.99$ & 45.9 & Asiago $122+\mathrm{BC}$ & 700 & $3 \times 1800$ \\
\hline 2017 Oct-21 & $58,047.26$ & 48.2 & $\mathrm{NTT}+\mathrm{EFOSC} 2$ & 200 & 900 \\
\hline 2017 Oct-22 & $58,048.54$ & 49.5 & FTS +FLOYDS & 500 & 2700 \\
\hline 2017 Oct-24 & $58,050.10$ & 51.0 & VLT+FORS2 & 500 & $2 \times 299$ \\
\hline 2017 Oct-27 & $58,053.52$ & 54.4 & FTS+FLOYDS & 500 & 2700 \\
\hline 2017 Oct-27 & $58,053.70$ & 54.6 & $\mathrm{MMT}+\mathrm{BCH}$ & 3900 & $3 \times 300$ \\
\hline 2017 Oct-28 & $58,054.37$ & 55.3 & $\mathrm{Bok}+\mathrm{BC}$ & 700 & $3 \times 240$ \\
\hline 2017 Oct-29 & $58,055.28$ & 56.3 & HET+LRS2B & 1100 & 1000 \\
\hline 2017 Nov 1 & $58,058.45$ & 59.4 & FTS+FLOYDS & 500 & 2700 \\
\hline 2017 Nov 2 & $58,059.80$ & 60.7 & $\mathrm{HCT}+\mathrm{HFOSC}$ & 350 & $2 \times 1800$ \\
\hline 2017 Nov 3 & $58,060.66$ & 61.6 & $\mathrm{HCT}+\mathrm{HFOSC}$ & 350 & $2 \times 1800$ \\
\hline 2017 Nov 5 & $58,062.79$ & 63.7 & $\mathrm{HCT}+\mathrm{HFOSC}$ & 350 & $2 \times 1200$ \\
\hline 2017 Nov 6 & $58,063.48$ & 64.4 & FTN+FLOYDS & 500 & 2700 \\
\hline 2017 Nov 6 & $58,063.79$ & 64.7 & $\mathrm{HCT}+\mathrm{HFOSC}$ & 350 & $2 \times 1200$ \\
\hline 2017 Nov 10 & $58,067.09$ & 68.0 & $\mathrm{NOT}+\mathrm{ALFOSC}$ & 300 & $2 \times 300$ \\
\hline 2017 Nov 10 & $58,067.24$ & 68.2 & VLT+FORS2 & 500 & 329 \\
\hline 2017 Nov 10 & $58,067.79$ & 68.7 & $\mathrm{HCT}+\mathrm{HFOSC}$ & 350 & $2 \times 1200$ \\
\hline 2017 Nov 13 & $58,070.63$ & 71.5 & $\mathrm{BAO}+\mathrm{BFOSC}$ & 500 & 3000 \\
\hline 2017 Nov 19 & $58,076.39$ & 77.3 & FTN+FLOYDS & 500 & 1800 \\
\hline 2017 Nov 19 & $58,076.73$ & 77.6 & $\mathrm{HCT}+\mathrm{HFOSC}$ & 350 & $2 \times 1800$ \\
\hline 2017 Nov 20 & $58,077.31$ & 78.2 & $\mathrm{MMT}+\mathrm{BCH}$ & 3900 & $3 \times 300$ \\
\hline 2017 Nov 23 & $58,080.72$ & 81.6 & $\mathrm{HCT}+\mathrm{HFOSC}$ & 350 & $2 \times 1200$ \\
\hline 2017 Nov 26 & $58,083.95$ & 84.9 & Asiago $122+\mathrm{BC}$ & 700 & $4 \times 1800$ \\
\hline 2017 Nov 29 & $58,086.57$ & 87.5 & $\mathrm{HCT}+\mathrm{HFOSC}$ & 350 & $2 \times 1200$ \\
\hline 2017 Dec 2 & $58,089.60$ & 90.5 & $\mathrm{HCT}+\mathrm{HFOSC}$ & 350 & $2 \times 1800$ \\
\hline 2017 Dec 3 & $58,090.91$ & 91.8 & Asiago $122+\mathrm{BC}$ & 700 & $4 \times 1800$ \\
\hline 2017 Dec 4 & $58,091.38$ & 92.3 & FTN+FLOYDS & 500 & 1800 \\
\hline 2017 Dec 7 & $58,094.04$ & 94.9 & $\mathrm{NOT}+\mathrm{ALFOSC}$ & 300 & 600 \\
\hline
\end{tabular}


Table 4

(Continued)

\begin{tabular}{|c|c|c|c|c|c|}
\hline $\begin{array}{l}\text { UT Date } \\
\text { (y m d) }\end{array}$ & MJD & $\begin{array}{l}\text { Phase } \\
\text { (days) }\end{array}$ & $\begin{array}{c}\text { Telescope+ } \\
\text { Instrument }\end{array}$ & $\begin{array}{c}R \\
\lambda / \Delta_{\lambda}\end{array}$ & $\begin{array}{l}\text { Exposure Time } \\
\text { (s) }\end{array}$ \\
\hline 2017 Dec 9 & $58,096.83$ & 97.7 & Asiago $122+B C$ & 700 & $4 \times 1800$ \\
\hline 2017 Dec 10 & $58,097.26$ & 98.2 & $\mathrm{Bok}+\mathrm{BC}$ & 700 & $3 \times 1200$ \\
\hline 2017 Dec 12 & $58,099.15$ & 100.1 & VLT+FORS2 & 500 & $2 \times 329$ \\
\hline 2017 Dec 13 & $58,100.12$ & 101.0 & VLT+FORS2 & 500 & 329 \\
\hline 2017 Dec 14 & $58,101.04$ & 102.0 & $\mathrm{VLT}+\mathrm{FORS} 2$ & 500 & 329 \\
\hline 2017 Dec 15 & $58,102.46$ & 103.4 & FTS+FLOYDS & 500 & 3600 \\
\hline 2017 Dec 18 & $58,105.8$ & 106.7 & Asiago $122+\mathrm{BC}$ & 700 & $4 \times 1800$ \\
\hline 2017 Dec 20 & $58,107.8$ & 108.7 & Asiago $122+\mathrm{BC}$ & 700 & $4 \times 1800$ \\
\hline 2017 Dec 21 & $58,108.11$ & 109.0 & VLT+FORS2 & 500 & $3 \times 329$ \\
\hline 2017 Dec 21 & $58,108.55$ & 109.5 & $\mathrm{BAO}+\mathrm{BFOSC}$ & 500 & 3000 \\
\hline 2017 Dec 22 & $58,109.11$ & 110.0 & VLT+FORS2 & 500 & $2 \times 329$ \\
\hline 2017 Dec 23 & $58,109.8$ & 110.8 & Asiago $122+\mathrm{BC}$ & 700 & $4 \times 1800$ \\
\hline 2017 Dec 23 & $58,110.04$ & 111.0 & $\mathrm{VLT}+\mathrm{FORS} 2$ & 500 & 329 \\
\hline 2017 Dec 23 & $58,110.23$ & 111.1 & $\mathrm{MMT}+\mathrm{BCH}$ & 3900 & $3 \times 300$ \\
\hline 2017 Dec 29 & $58,116.59$ & 117.5 & $\mathrm{HCT}+\mathrm{HFOSC}$ & 350 & $2 \times 1500$ \\
\hline 2018 Jan 5 & $58,123.45$ & 124.4 & FTS+FLOYDS & 500 & 3600 \\
\hline 2018 Jan 7 & $58,126.42$ & 127.3 & $\mathrm{BAO}+\mathrm{BFOSC}$ & 500 & 3000 \\
\hline 2018 Jan 9 & $58,128.82$ & 129.7 & Asiago182+AFOSC & 300 & $2 \times 1200$ \\
\hline 2018 Jan-14 & $58,132.05$ & 133.0 & $\mathrm{VLT}+\mathrm{FORS} 2$ & 500 & 329 \\
\hline 2018 Jan-14 & $58,132.56$ & 133.5 & $\mathrm{HCT}+\mathrm{HFOSC}$ & 350 & $2 \times 1500$ \\
\hline 2018 Jan-15 & $58,133.07$ & 134.0 & $\mathrm{VLT}+\mathrm{FORS} 2$ & 500 & 329 \\
\hline 2018 Jan-16 & $58,134.12$ & 135.0 & VLT+FORS2 & 500 & 329 \\
\hline 2018 Jan-16 & $58,134.26$ & 135.2 & FTN+FLOYDS & 500 & 3600 \\
\hline 2018 Jan-17 & $58,135.05$ & 136.0 & VLT + FORS2 & 500 & 329 \\
\hline 2018 Jan-18 & $58,136.05$ & 137.0 & $\mathrm{VLT}+\mathrm{FORS} 2$ & 500 & 329 \\
\hline 2018 Jan-17 & $58,136.46$ & 137.4 & $\mathrm{BAO}+\mathrm{BFOSC}$ & 500 & 3300 \\
\hline 2018 Jan-19 & $58,137.07$ & 138.0 & VLT+FORS2 & 500 & $2 \times 329$ \\
\hline 2018 Jan-20 & $58,137.89$ & 138.8 & $\mathrm{NOT}+\mathrm{ALFOSC}$ & 300 & 600 \\
\hline 2018 Jan-22 & $58,141.47$ & 142.4 & $\mathrm{BAO}+\mathrm{BFOSC}$ & 500 & 3000 \\
\hline 2018 Jan-24 & $58,142.15$ & 143.1 & $\mathrm{Bok}+\mathrm{BC}$ & 700 & $3 \times 600$ \\
\hline 2018 Feb 5 & $58,154.09$ & 155.0 & $\mathrm{MMT}+\mathrm{BCH}$ & 3900 & $3 \times 900$ \\
\hline 2018 Feb 12 & $58,161.22$ & 162.1 & FTN+FLOYDS & 500 & 3600 \\
\hline 2018 Jan 20 & $58,163.86$ & 164.7 & $\mathrm{NOT}+\mathrm{ALFOSC}$ & 300 & 600 \\
\hline 2018 Jul 12 & $58,311.37$ & 312.3 & Magellan+MIKE & 40000 & $3 \times 1200$ \\
\hline
\end{tabular}

Note. Phases are reported with respect to an explosion epoch of 57999.09.

and South at Haleakala, USA, and Siding Spring, Australia, respectively, through the Global Supernova Project. A $2^{\prime \prime}$ slit was placed on the target at the parallactic angle. Onedimensional spectra were extracted, reduced, and calibrated following standard procedures using the FLOYDS pipeline (Valenti et al. 2014). HIRES spectra were reduced using the MAuna Kea Echelle Extraction (MAKEE) data reduction package $^{53}$ (written by T. Barlow). MIKE spectra were reduced using the latest version of the MIKE pipeline ${ }^{54}$ (written by D. Kelson).

\section{B.2. NIR Spectroscopy}

A sequence of NIR spectra of SN $2017 \mathrm{gmr}$ were also taken and are logged in Table 5. All NIR spectra were taken using a classical ABBA technique, dithering the object along the slit in order to facilitate good sky subtraction. Further, the slit was oriented along the parallactic angle to minimize slit losses due to atmospheric differential refraction (Filippenko 1982). In all cases, an A0V star was observed either before or after the science observations in order to correct for telluric absorption

\footnotetext{
53 http://spider.ipac.caltech.edu/staff/tab/makee/

54 http://code.obs.carnegiescience.edu/mike/
}

and flux-calibrate the data, following the prescriptions of Vacca et al. (2003).

Gemini/GNIRS data were taken in cross-dispersed mode with the 0 ". 675 slit, yielding continuous wavelength coverage from 0.8 to $2.5 \mu \mathrm{m}$ and an $R \sim 1000$. These data were reduced with the XDGNIRS pipeline provided by Gemini Observatory, as described in Sand et al. (2016) and Hsiao et al. (2019).

IRTF spectra were taken in SXD mode and the 0".5 slit, yielding wavelength coverage from $\sim 0.8$ to $2.4 \mu \mathrm{m}$ and $R \sim 1200$. These data were reduced using the publicly available Spextool software package (Cushing et al. 2004), as described in Hsiao et al. (2019).

Two NIR spectra were taken with the Son OF ISAAC (SOFI) spectrograph mounted on the NTT telescope (Moorwood et al. 1998), using both the Blue and Red grisms, giving a broad wavelength coverage of $0.9-2.4 \mu \mathrm{m}$. The SOFI spectra were taken as part of the ePESSTO program and were reduced as described in Smartt et al. (2015).

Finally, a single FIRE spectrum was taken using the high throughput prism mode with a 0 "! 6 slit, giving continuous wavelength coverage from 0.8 to $2.5 \mu \mathrm{m}$ and a resolution of $R \sim 500$ in the $J$ band. The spectrum was reduced with the purpose-built FIREHOSE pipeline (Simcoe et al. 2013) as described in detail in Hsiao et al. (2019). 
Table 5

NIR Spectroscopy of SN 2017gmr

\begin{tabular}{|c|c|c|c|c|c|}
\hline $\begin{array}{l}\text { UT Date } \\
\text { (y m d) }\end{array}$ & MJD & Phase & $\begin{array}{l}\text { Telescope } \\
\text { Instrument }\end{array}$ & $\begin{array}{c}\lambda \text { Range } \\
(\mu \mathrm{m})\end{array}$ & $\begin{array}{c}\text { Exp Time } \\
\text { (s) }\end{array}$ \\
\hline 2017 Sep 6 & $58,002.46$ & +3.4 & Gemini/GNIRS & $0.82-2.4$ & $14 \times 120$ \\
\hline 2017 Sep 10 & $58,005.57$ & +6.5 & IRTF/SpeX & $0.82-2.4$ & $8 \times 150$ \\
\hline 2017 Oct 20 & $58,046.17$ & +47.1 & NTT/SOFI & $0.9-2.4$ & $12 \times 125$ \\
\hline 2017 Oct 28 & $58,054.31$ & +54.2 & IRTF/SpeX & $0.82-2.4$ & $12 \times 150$ \\
\hline 2017 Nov 28 & $58,085.14$ & +86.1 & NTT/SOFI & $0.9-2.4$ & $8 \times 120$ \\
\hline 2018 Jan 31 & $58,149.02$ & +149.9 & Magellan/FIRE & $0.8-2.4$ & $12 \times 127$ \\
\hline
\end{tabular}

Note. Phase calculated with respect to our assumed explosion epoch, MJD $=57,999.09$.

\section{ORCID iDs}

Jennifer E. Andrews (iD https://orcid.org/0000-00030123-0062

D. J. Sand (ib https://orcid.org/0000-0003-4102-380X

S. Valenti (iD https://orcid.org/0000-0001-8818-0795

Raya Dastidar (iD https://orcid.org/0000-0001-6191-7160

Kuntal Misra (i) https://orcid.org/0000-0003-1637-267X

Avinash Singh (D) https://orcid.org/0000-0003-2091-622X

D. Hiramatsu (iD https://orcid.org/0000-0002-1125-9187

P. J. Brown (1) https://orcid.org/0000-0001-6272-5507

G. Hosseinzadeh (iD https://orcid.org/0000-0002-0832-2974

J. Vinko (D) https://orcid.org/0000-0001-8764-7832

I. Arcavi (iD https://orcid.org/0000-0001-7090-4898

Chris Ashall (i) https://orcid.org/0000-0002-5221-7557

Marco Berton (1) https://orcid.org/0000-0002-1058-9109

K. A. Bostroem (1) https://orcid.org/0000-0002-4294-444X

L. Chomiuk (ib https://orcid.org/0000-0002-8400-3705

Morgan Fraser (i) https://orcid.org/0000-0003-2191-1674

L. Galbany (ib https://orcid.org/0000-0002-1296-6887

C. Gall (i) https://orcid.org/0000-0002-8526-3963

A. Gal-Yam (iD https://orcid.org/0000-0002-3653-5598

D. A. Howell (i) https://orcid.org/0000-0003-4253-656X

E. Y. Hsiao (iD https://orcid.org/0000-0003-1039-2928

C. Inserra (i) https://orcid.org/0000-0002-3968-4409

E. Kankare (i) https://orcid.org/0000-0001-8257-3512

H. Kuncarayakti (i) https://orcid.org/0000-0002-1132-1366

Brajesh Kumar (iD https://orcid.org/0000-0001-7225-2475

K. Maguire (iD https://orcid.org/0000-0002-9770-3508

P. Mazzali (1) https://orcid.org/0000-0001-6876-8284

C. McCully (i) https://orcid.org/0000-0001-5807-7893

P. Milne (1) https://orcid.org/0000-0002-0370-157X

N. Morrell (1) https://orcid.org/0000-0003-2535-3091

M. Nicholl (1) https://orcid.org/0000-0002-2555-3192

F. Patat (i) https://orcid.org/0000-0002-0537-3573

M. Phillips (i) https://orcid.org/0000-0003-2734-0796

G. Pignata (ib https://orcid.org/0000-0003-0006-0188

A. Reguitti (1) https://orcid.org/0000-0003-4254-2724

D. E. Reichart (iD https://orcid.org/0000-0002-5060-3673

Ó. Rodríguez (iD https://orcid.org/0000-0001-8651-8772

M. Shahbandeh (i) https://orcid.org/0000-0002-9301-5302

S. Smartt (ib https://orcid.org/0000-0002-8229-1731

J. Strader (iD https://orcid.org/0000-0002-1468-9668

M. D. Stritzinger (iD https://orcid.org/0000-0002-5571-1833

R. Szakáts (iD https://orcid.org/0000-0002-1698-605X

L. Tartaglia (i) https://orcid.org/0000-0003-3433-1492

Lingzhi Wang (iD https://orcid.org/0000-0002-1094-3817
Xiaofeng Wang (iD https://orcid.org/0000-0002-7334-2357

J. C. Wheeler (ib https://orcid.org/0000-0003-1349-6538

D. R. Young (iD https://orcid.org/0000-0002-1229-2499

\section{References}

Anand, G. S., Rizzi, L., \& Tully, R. B. 2018, AJ, 156, 105

Anderson, J. P. 2019, A\&A, 628, A7

Anderson, J. P., González-Gaitán, S., Hamuy, M., et al. 2014, ApJ, 786, 67 Andrews, J. E., Gallagher, J. S., Clayton, G. C., et al. 2010, ApJ, 715, 541

Andrews, J. E., Krafton, K. M., Clayton, G. C., et al. 2016, MNRAS, 457, 3241

Andrews, J. E., \& Smith, N. 2018, MNRAS, 477, 74

Andrews, J. E., Smith, N., McCully, C., et al. 2017, MNRAS, 471, 4047

Appenzeller, I., Fricke, K., Fürtig, W., et al. 1998, Msngr, 94, 1

Arcavi, I. 2017, in Hydrogen-Rich Core-Collapse Supernovae, ed. A. W. Alsabti \& P. Murdin (Berlin: Springer), 239

Astropy Collaboration, Robitaille, T. P., Tollerud, E. J., et al. 2013, A\&A, 558, A33

Beasor, E. R., \& Davies, B. 2018, MNRAS, 475, 55

Benetti, S., Patat, F., Turatto, M., et al. 1994, A\&A, 285, L13

Bernstein, R., Shectman, S. A., Gunnels, S. M., Mochnacki, S., \& Athey, A. E. 2003, Proc. SPIE, 4841, 1694

Bevan, A., Wesson, R., Barlow, M. J., et al. 2019, MNRAS, 485, 5192

Bilinski, C., Smith, N., Li, W., et al. 2015, MNRAS, 450, 246

Bose, S., Dong, S., Elias-Rosa, N., et al. 2019, ApJL, 873, L3

Bose, S., Sutaria, F., Kumar, B., et al. 2015a, ApJ, 806, 160

Bose, S., Valenti, S., Misra, K., et al. 2015b, MNRAS, 450, 2373

Branch, D., \& Wheeler, J. C. 2017, Supernova Explosions (Berlin: Springer)

Breeveld, A. A., Landsman, W., Holland, S. T., et al. 2011, in AIP Conf. Ser.

1358, Gamma Ray Bursts, ed. J. E. McEnery, J. L. Racusin, \& N. Gehrels (New York: AIP), 373

Brown, P. J., Breeveld, A. A., Holland, S., Kuin, P., \& Pritchard, T. 2014, Ap\&SS, 354, 89

Brown, P. J., Holland, S. T., Immler, S., et al. 2009, AJ, 137, 4517

Brown, T. M., Baliber, N., Bianco, F. B., et al. 2013, PASP, 125, 1031

Bullivant, C., Smith, N., Williams, G. G., et al. 2018, MNRAS, 476, 1497

Cardelli, J. A., Clayton, G. C., \& Mathis, J. S. 1989, ApJ, 345, 245

Chonis, T. S., Hill, G. J., Lee, H., et al. 2016, Proc. SPIE, 9908, 99084C

Chugai, N. N., Fabrika, S. N., Sholukhova, O. N., et al. 2005, AstL, 31, 792

Clemens, J. C., Crain, J. A., \& Anderson, R. 2004, Proc. SPIE, 5492, 331

Cushing, M. C., Vacca, W. D., \& Rayner, J. T. 2004, PASP, 116, 362

Dastidar, R., Misra, K., Hosseinzadeh, G., et al. 2018, MNRAS, 479, 2421

Dastidar, R., Misra, K., Singh, M., et al. 2019, MNRAS, 486, 2850

Davies, B., \& Dessart, L. 2019, MNRAS, 483, 887

de Jaeger, T., Anderson, J. P., Galbany, L., et al. 2018a, MNRAS, 476, 4592 de Jaeger, T., Galbany, L., Gutiérrez, C. P., et al. 2018b, MNRAS, 478, 3776 de Jaeger, T., González-Gaitán, S., Hamuy, M., et al. 2017, ApJ, 835, 166 de Mink, S. E., Sana, H., Langer, N., Izzard, R. G., \& Schneider, F. R. N. 2014, ApJ, 782, 7

Dessart, L., \& Hillier, D. J. 2005a, A\&A, 437, 667

Dessart, L., \& Hillier, D. J. 2005b, A\&A, 439, 671

Dessart, L., \& Hillier, D. J. 2011, MNRAS, 415, 3497

Dressler, A., Bigelow, B., Hare, T., et al. 2011, PASP, 123, 288

Elias, J. H., Joyce, R. R., Liang, M., et al. 2006, Proc. SPIE, 6269, 62694C 
Elias-Rosa, N., Pastorello, A., Benetti, S., et al. 2016, MNRAS, 463, 3894 Elias-Rosa, N., Pursimo, T., Korhonen, H., et al. 2017, ATel, 10746, 1 Elmhamdi, A., Chugai, N. N., \& Danziger, I. J. 2003a, A\&A, 404, 1077 Elmhamdi, A., Danziger, I. J., Chugai, N., et al. 2003b, MNRAS, 338, 939 Faran, T., Poznanski, D., Filippenko, A. V., et al. 2014, MNRAS, 442, 844 Filippenko, A. V. 1982, PASP, 94, 715

Förster, F., Moriya, T. J., Maureira, J. C., et al. 2018, NatAs, 2, 808 Fox, O. D., Chevalier, R. A., Dwek, E., et al. 2010, ApJ, 725, 1768 Fraser, M., Magee, M., Kotak, R., et al. 2013, ApJL, 779, L8 Fuller, J. 2017, MNRAS, 470, 1642

Galbany, L., Hamuy, M., Phillips, M. M., et al. 2016, AJ, 151, 33

Gall, C., Hjorth, J., Watson, D., et al. 2014, Natur, 511, 326

Gall, E. E. E., Kotak, R., Leibundgut, B., et al. 2018, A\&A, 611, A25

Gal-Yam, A. 2017, in Observational and Physical Classification of Supernovae, ed. A. W. Alsabti \& P. Murdin (Berlin: Springer), 195 Gal-Yam, A., Arcavi, I., Ofek, E. O., et al. 2014, Natur, 509, 471 Gal-Yam, A., \& Leonard, D. C. 2009, Natur, 458, 865

Gal-Yam, A., Leonard, D. C., Fox, D. B., et al. 2007, ApJ, 656, 372 Gehrels, N., Chincarini, G., Giommi, P., et al. 2004, ApJ, 611, 1005 Gerardy, C. L., Fesen, R. A., Höflich, P., \& Wheeler, J. C. 2000, AJ, 119, 2968 Goldman, S. R., van Loon, J. T., Zijlstra, A. A., et al. 2017, MNRAS, 465, 403 González-Gaitán, S., Tominaga, N., Molina, J., et al. 2015, MNRAS, 451, 2212

Groh, J. H., Meynet, G., Ekström, S., \& Georgy, C. 2014, A\&A, 564, A30 Gutiérrez, C. P., Anderson, J. P., Hamuy, M., et al. 2017a, ApJ, 850, 89 Gutiérrez, C. P., Anderson, J. P., Hamuy, M., et al. 2017b, ApJ, 850, 90 Hamuy, M. 2003, ApJ, 582, 905

Hamuy, M. 2005, in IAU Coll. 192: Cosmic Explosions, On the X Anniversary of SN1993J, ed. J.-M. Marcaide \& K. W. Weiler (Cambridge: Cambridge Univ. Press), 535

Hamuy, M., \& Pinto, P. A. 2002, ApJL, 566, L63

Hamuy, M., Pinto, P. A., Maza, J., et al. 2001, ApJ, 558, 615

Hendry, M. A., Smartt, S. J., Maund, J. R., et al. 2005, MNRAS, 359, 906

Hoffman, J. L., Leonard, D. C., Chornock, R., et al. 2008, ApJ, 688, 1186

Hosseinzadeh, G. 2019, Light Curve Fitting v.0.0.0, Zenodo, doi:10.5281/ zenodo. 2639464

Hosseinzadeh, G., Valenti, S., McCully, C., et al. 2018, ApJ, 861, 63

Hsiao, E. Y., Phillips, M. M., Marion, G. H., et al. 2019, PASP, 131, 014002

Huang, F., Li, J.-Z., Wang, X.-F., et al. 2012, RAA, 12, 1585

Huang, F., Wang, X., Zampieri, L., et al. 2016, ApJ, 832, 139

Huang, F., Wang, X.-F., Hosseinzadeh, G., et al. 2018, MNRAS, 475, 3959

Inserra, C., Turatto, M., Pastorello, A., et al. 2011, MNRAS, 417, 261

Jerkstrand, A., Fransson, C., Maguire, K., et al. 2012, A\&A, 546, A28

Khazov, D., Yaron, O., Gal-Yam, A., et al. 2016, ApJ, 818, 3

Kilpatrick, C. D., Andrews, J. E., Smith, N., et al. 2016, MNRAS, 463, 1088

Kiminki, D. C., \& Kobulnicky, H. A. 2012, ApJ, 751, 4

Kirshner, R. P., \& Kwan, J. 1974, ApJ, 193, 27

Koribalski, B. S., Staveley-Smith, L., Kilborn, V. A., et al. 2004, AJ, 128, 16

Kotak, R., Meikle, W. P. S., Farrah, D., et al. 2009, ApJ, 704, 306

Leonard, D. C. 2007, ApJ, 670, 1275

Leonard, D. C., Filippenko, A. V., Barth, A. J., \& Matheson, T. 2000, ApJ, 536, 239

Leonard, D. C., Filippenko, A. V., Ganeshalingam, M., et al. 2006, Natur, 440,505

Leonard, D. C., Filippenko, A. V., Gates, E. L., et al. 2002, PASP, 114, 35

Levesque, E. M. 2017, Astrophysics of Red Supergiants (Bristol: Institute of Physics Publishing)

Lucy, L. B., Danziger, I. J., Gouiffes, C., \& Bouchet, P. 1989, in IAU Coll. 120: Structure and Dynamics of the Interstellar Medium, ed. G. Tenorio-Tagle, M. Moles, \& J. Melnick (Berlin: Springer), 164

Maeda, K., Kawabata, K., Mazzali, P. A., et al. 2008, Sci, 319, 1220

Maguire, K., Di Carlo, E., Smartt, S. J., et al. 2010, MNRAS, 404, 981

Mattila, S., Meikle, W. P. S., Lundqvist, P., et al. 2008, MNRAS, 389, 141

Mauerhan, J., \& Smith, N. 2012, MNRAS, 424, 2659

Mauerhan, J., Williams, G. G., Smith, N., et al. 2014, MNRAS, 442, 1166

Mauerhan, J. C., Smith, N., Filippenko, A. V., et al. 2013, MNRAS, 430, 1801

Mauron, N., \& Josselin, E. 2011, A\&A, 526, A156

Mazzali, P. A., Kawabata, K. S., Maeda, K., et al. 2005, Sci, 308, 1284

Moe, M., \& Di Stefano, R. 2017, ApJS, 230, 15

Montargès, M., Norris, R., Chiavassa, A., et al. 2018, A\&A, 614, A12

Moorwood, A., Cuby, J. G., Biereichel, P., et al. 1998, Msngr, 94, 7

Moriya, T. J., Förster, F., Yoon, S.-C., Gräfener, G., \& Blinnikov, S. I. 2018, MNRAS, 476, 2840

Morozova, V., Piro, A. L., \& Valenti, S. 2017, ApJ, 838, 28

Morozova, V., Piro, A. L., \& Valenti, S. 2018, ApJ, 858, 15

Müller, T., Prieto, J. L., Pejcha, O., \& Clocchiatti, A. 2017, ApJ, 841, 127
Nagao, T., Cikota, A., Patat, F., et al. 2019, MNRAS, 489, L69 Nakaoka, T., Kawabata, K. S., Maeda, K., et al. 2018, ApJ, 859, 78 Nicholl, M. 2018, RNAAS, 2, 230

Nicholl, M., Berger, E., Smartt, S. J., et al. 2016, ApJ, 826, 39 Niemela, V. S., Ruiz, M. T., \& Phillips, M. M. 1985, ApJ, 289, 52 Nugent, P., Sullivan, M., Ellis, R., et al. 2006, ApJ, 645, 841

Ofek, E. O., Sullivan, M., Cenko, S. B., et al. 2013, Natur, 494, 65 Ofek, E. O., Sullivan, M., Shaviv, N. J., et al. 2014, ApJ, 789, 104 Pejcha, O., \& Prieto, J. L. 2015, ApJ, 799, 215

Phillips, M. M., Simon, J. D., Morrell, N., et al. 2013, ApJ, 779, 38 Rodríguez, Ó., Pignata, G., Hamuy, M., et al. 2019, MNRAS, 483, 5459 Poznanski, D., Butler, N., Filippenko, A. V., et al. 2009, ApJ, 694, 1067 Poznanski, D., Prochaska, J. X., \& Bloom, J. S. 2012, MNRAS, 426, 1465 Prabhu, T. P., \& Anupama, G. C. 2010, BASI, 1, 193

Pursimo, T., Elias-Rosa, N., Dennefeld, M., et al. 2017, ATel, 10717, 1

Quimby, R. M., Wheeler, J. C., Höflich, P., et al. 2007, ApJ, 666, 1093

Rayner, J. T., Toomey, D. W., Onaka, P. M., et al. 2003, PASP, 115, 362

Reguitti, A., Pastorello, A., Pignata, G., et al. 2019, MNRAS, 482, 2750

Reichart, D., Nysewander, M., Moran, J., et al. 2005, NCimC, 28, 767

Rho, J., Geballe, T. R., Banerjee, D. P. K., et al. 2018, ApJL, 864, L20

Riess, A. G., Casertano, S., Yuan, W., et al. 2018, ApJ, 861, 126

Rubin, A., \& Gal-Yam, A. 2017, ApJ, 848, 8

Rubin, A., Gal-Yam, A., De Cia, A., et al. 2016, ApJ, 820, 33

Rui, L., Wang, X., Mo, J., et al. 2019, MNRAS, 485, 1990

Sagar, R. 1999, CSci, 77, 643

Sagar, R., Kumar, B., Omar, A., \& Joshi, Y. C. 2012, BASI, 4, 173

Sahu, D. K., Anupama, G. C., Srividya, S., \& Muneer, S. 2006, MNRAS, 372,1315

Sana, H., de Mink, S. E., de Koter, A., et al. 2012, Sci, 337, 444

Sand, D. J., Hsiao, E. Y., Banerjee, D. P. K., et al. 2016, ApJL, 822, L16

Sapir, N., \& Waxman, E. 2017, ApJ, 838, 130

Sarangi, A., \& Cherchneff, I. 2013, ApJ, 776, 107

Sarangi, A., Matsuura, M., \& Micelotta, E. R. 2018, SSRv, 214, 63

Schlafly, E. F., \& Finkbeiner, D. P. 2011, ApJ, 737, 103

Schlegel, E. M. 1990, MNRAS, 244, 269

SDSS Collaboration, Albareti, F. D., Allende Prieto, C., et al. 2017, ApJS, 233, 25

Shiode, J. H., \& Quataert, E. 2014, ApJ, 780, 96

Shivvers, I., Groh, J. H., Mauerhan, J. C., et al. 2015, ApJ, 806, 213

Simcoe, R. A., Burgasser, A. J., Schechter, P. L., et al. 2013, PASP, 125, 270

Singh, A., Srivastav, S., Kumar, B., Anupama, G. C., \& Sahu, D. K. 2018, MNRAS, 480, 2475

Smartt, S. J., Eldridge, J. J., Crockett, R. M., \& Maund, J. R. 2009, MNRAS, 395, 1409

Smartt, S. J., Valenti, S., Fraser, M., et al. 2015, A\&A, 579, A40

Smith, N. 2014, ARA\&A, 52, 487

Smith, N. 2017, in Interacting Supernovae: Types IIn and Ibn, ed. A. W. Alsabti \& P. Murdin (Berlin: Springer), 403

Smith, N., \& Arnett, W. D. 2014, ApJ, 785, 82

Smith, N., Cenko, S. B., Butler, N., et al. 2012a, MNRAS, 420, 1135

Smith, N., Foley, R. J., \& Filippenko, A. V. 2008, ApJ, 680, 568

Smith, N., Li, W., Filippenko, A. V., \& Chornock, R. 2011a, MNRAS, 412, 1522

Smith, N., Li, W., Silverman, J. M., Ganeshalingam, M., \& Filippenko, A. V. 2011b, MNRAS, 415, 773

Smith, N., Mauerhan, J. C., Cenko, S. B., et al. 2015, MNRAS, 449, 1876

Smith, N., Silverman, J. M., Chornock, R., et al. 2009, ApJ, 695, 1334

Smith, N., Silverman, J. M., Filippenko, A. V., et al. 2012b, AJ, 143, 17

Stetson, P. B. 1987, PASP, 99, 191

Stetson, P. B. 2000, PASP, 112, 925

Stritzinger, M., Taddia, F., Fransson, C., et al. 2012, ApJ, 756, 173

Strotjohann, N. L., Ofek, E. O., Gal-Yam, A., et al. 2015, ApJ, 811, 117

Sugerman, B. E. K., Ercolano, B., Barlow, M. J., et al. 2006, Sci, 313, 196

Szalai, T., Vinkó, J., Könyves-Tóth, R., et al. 2019, ApJ, 876, 19

Takáts, K., \& Vinkó, J. 2006, MNRAS, 372, 1735

Tartaglia, L., Pastorello, A., Sullivan, M., et al. 2016, MNRAS, 459, 1039

Tartaglia, L., Sand, D. J., Valenti, S., et al. 2018, ApJ, 853, 62

Taubenberger, S., Valenti, S., Benetti, S., et al. 2009, MNRAS, 397, 677

Terreran, G., Jerkstrand, A., Benetti, S., et al. 2016, MNRAS, 462, 137

The Astropy Collaboration, Price-Whelan, A. M., Sipőcz, B. M., et al. 2018, AJ, 156, 123

Tinyanont, S., Kasliwal, M. M., Krafton, K., et al. 2019, ApJ, 873, 127 Tomasella, L., Cappellaro, E., Fraser, M., et al. 2013, MNRAS, 434, 1636 Turatto, M. 2003, in Supernovae and Gamma-Ray Bursters, ed. K. Weiler (Berlin: Springer), 21

Vacca, W. D., Cushing, M. C., \& Rayner, J. T. 2003, PASP, 115, 389 
Valenti, S., Howell, D. A., Stritzinger, M. D., et al. 2016, MNRAS, 459, 3939

Valenti, S., Sand, D., Pastorello, A., et al. 2014, MNRAS, 438, L101

Valenti, S., Tartaglia, L., Sand, D., et al. 2017, ATel, 10706, 1

Van Dyk, S. D., Li, W., \& Filippenko, A. V. 2003, PASP, 115, 1

Van Dyk, S. D., Zheng, W., Maund, J. R., et al. 2019, ApJ, 875, 136

Vinkó, J., Sárneczky, K., Takáts, K., et al. 2012, A\&A, 546, A12

Vinkó, J., Takáts, K., Sárneczky, K., et al. 2006, MNRAS, 369, 1780

Vogt, S. S., Allen, S. L., Bigelow, B. C., et al. 1994, Proc. SPIE, 2198, 362
Williams, G. G., Milne, P. A., Park, H. S., et al. 2008, in AIP Conf. Ser. 1000, Gamma Ray Bursts, ed. M. Galassi, D. Palmer, \& E. Fenimore (New York: AIP), 535

Wittkowski, M., Arroyo-Torres, B., Marcaide, J. M., et al. 2017, A\&A, 597, A9

Woosley, S. E., Pinto, P. A., \& Hartmann, D. 1989, ApJ, 346, 395

Yaron, O., \& Gal-Yam, A. 2012, PASP, 124, 668

Yaron, O., Perley, D. A., Gal-Yam, A., et al. 2017, NatPh, 13, 510

Yuan, F., Jerkstrand, A., Valenti, S., et al. 2016, MNRAS, 461, 2003 